\title{
Electron Pre-acceleration at Nonrelativistic High-Mach-number Perpendicular Shocks
}

\author{
Artem Bohdan ${ }^{1}$ (1) Jacek Niemiec $^{1}$ (1), Oleh $\operatorname{Kobzar}^{1}$ (1), and Martin Pohl ${ }^{2,3}$ (1) \\ ${ }^{1}$ Instytut Fizyki Jạdrowej PAN, ul. Radzikowskiego 152, 31-342 Kraków, Poland; artem.bohdan@ifj.edu.pl \\ ${ }^{2}$ Institute of Physics and Astronomy, University of Potsdam, D-14476 Potsdam, Germany \\ DESY, D-15738 Zeuthen, Germany \\ Received 2017 May 31; revised 2017 August 4; accepted 2017 August 16; published 2017 September 21
}

\begin{abstract}
We perform particle-in-cell simulations of perpendicular nonrelativistic collisionless shocks to study electron heating and pre-acceleration for parameters that permit the extrapolation to the conditions at young supernova remnants. Our high-resolution large-scale numerical experiments sample a representative portion of the shock surface and demonstrate that the efficiency of electron injection is strongly modulated with the phase of the shock reformation. For plasmas with low and moderate temperature (plasma beta $\beta_{\mathrm{p}}=5 \cdot 10^{-4}$ and $\beta_{\mathrm{p}}=0.5$ ), we explore the nonlinear shock structure and electron pre-acceleration for various orientations of the large-scale magnetic field with respect to the simulation plane, while keeping it at $90^{\circ}$ to the shock normal. Ion reflection off of the shock leads to the formation of magnetic filaments in the shock ramp, resulting from Weibel-type instabilities, and electrostatic Buneman modes in the shock foot. In all of the cases under study, the latter provides first-stage electron energization through the shock-surfing acceleration mechanism. The subsequent energization strongly depends on the field orientation and proceeds through adiabatic or second-order Fermi acceleration processes for configurations with the out-of-plane and in-plane field components, respectively. For strictly out-of-plane field, the fraction of suprathermal electrons is much higher than for other configurations, because only in this case are the Buneman modes fully captured by the 2D simulation grid. Shocks in plasma with moderate $\beta_{\mathrm{p}}$ provide more efficient pre-acceleration. The relevance of our results to the physics of fully 3D systems is discussed.
\end{abstract}

Key words: acceleration of particles - instabilities - ISM: supernova remnants - methods: numerical - plasmas shock

\section{Introduction}

The acceleration of charged particles is a key topic in astrophysical research. High-energy particles are found at astrophysical and interplanetary shocks, and collisionless nonrelativistic shocks of supernova remnants (SNRs) are widely believed to be the sources of galactic cosmic rays (CRs) with energies up to the knee at $\sim 10^{15} \mathrm{eV}$. Detection of high-energy $\gamma$-ray emission from SNRs by satellite and groundbased observatories prove the energetic particle production in these sources, although it is generally unclear whether the observed nonthermal emission comes primarily from protons or electrons (Aharonian et al. 2007).

A dominant particle acceleration mechanism assumed to operate at nonrelativistic shock waves is diffusive shock acceleration (DSA), a first-order Fermi process (e.g., Drury 1983; Blandford \& Eichler 1987). In this process particles gain their energies in repetitive interactions with the shock front. They are confined to the shock vicinity by magnetohydrodynamic (MHD) inhomogeneities that elastically scatter the particles in pitch-angle, providing for their diffusive motion in the upstream and downstream regions of the shock. The most tantalizing unresolved question in DSA theory is the so-called particle injection problem: DSA works only for particles with a Larmor radius that is larger than the width of the shock transition layer, which is typically of the order of several proton gyroradii. Some pre-acceleration is thus required, in particular for electrons, on account of their lower mass and consequently smaller Larmor radii and inertial lengths, than compared to protons.

Here we study perpendicular shocks in a regime of high Alfvénic and sonic Mach numbers, $M_{\mathrm{A}} \gtrsim 30$ and $M_{\mathrm{s}} \gtrsim 50$, as appropriate for forward shocks of young SNRs. Using hybrid simulations, ion pre-acceleration has been shown to be inefficient at perpendicular shocks (e.g., Caprioli \& Spitkovsky 2014), and we focus on electron pre-acceleration. The physics of such shocks is governed by ion reflection at the shock ramp, and a variety of instabilities can be excited in the foot region due to the interaction of upstream plasma with reflected ions (Treumann 2009). The electrostatic two-stream instability, also known as Buneman instability (Buneman 1958), is a result of the interaction between cold incoming electrons and reflected ions. Early 1D simulations (Hoshino \& Shimada 2002) showed that the Buneman instability plays an important role in the generation of suprathermal electrons via shock surfing acceleration (SSA). Strong electrostatic waves are observed as a result of the nonlinear evolution of the Buneman instability. While electrons are captured in the electrostatic potential wells, they can be accelerated in a perpendicular direction by the convective electric field. Multiple rapid interactions of electrons with electrostatic waves in the foot region of a shock give rise to SSA and produce suprathermal tails in the electron spectra. 2D simulations of SSA at perpendicular shocks have been discussed in a number of papers (e.g., Amano \& Hoshino 2009a; Matsumoto et al. 2012, 2013; Wieland et al. 2016). The trajectories of accelerated electrons in multidimensional systems are more complicated than in $1 \mathrm{D}$, but the process is still referred to as SSA. Our understanding of the efficiency of SSA and its dependence on the ion-to-electron mass ratio, the plasma temperature, the magnetic-field orientation with respect to the simulation plane, etc., is still incomplete.

Since the Buneman instability is an electron-scale phenomenon, electron scales need to be resolved in simulations. We conduct particle-in-cell (PIC) simulations in 2D3V configuration, meaning that we follow two spatial coordinates and all 
three components of velocity and electromagnetic fields. In contrast to hybrid simulations, PIC simulations follow electron trajectories as well as the ion dynamics.

Our simulations complement previous 2D shock investigations (e.g., Matsumoto et al. 2012, 2013, 2015; Wieland et al. 2016). To explore electron energization via SSA, the Alfvénic Mach number, $M_{\mathrm{A}}$, should satisfy a threshold condition for the excitation of electrostatic waves in the shock foot (Matsumoto et al. 2012). The thermal velocity of the electrons should be smaller than the relative speed between incoming electrons and reflected ions, leading to (Wieland et al. 2016):

$$
M_{s} \gtrsim \frac{1+\alpha}{2} \sqrt{\frac{m_{i}}{m_{e}}} \sqrt{\frac{T_{e}}{T_{i}}},
$$

where $\alpha \simeq 0.2$ is the density ratio of reflected to incoming ions. Note that the ion temperature, $T_{i}$, is measured far upstream, whereas the electron temperature, $T_{e}$, is local, because the threshold condition involves the local thermal speed of electrons. If the electron gyrofrequency is substantially lower than the plasma frequency $\left(\Omega_{e} \simeq 0.1 \omega_{\text {pe }}\right.$ in our simulations), the growth rate and wave vector of the Buneman mode are only weakly affected by the magnetic field, even if that is oriented perpendicular to the wave vector (Strangeway 1982; Bohata et al. 2011).

In addition to the instability condition for the Buneman modes, the electrostatic potential should be strong enough to trap electrons and hold them during acceleration. Matsumoto et al. (2012) used an estimate of the energy that is transferred from the incoming electrons to Buneman waves to find the balance between the trapping force of the Buneman waves at saturation level and the Lorentz force for escaping, which leads to the relation:

$$
M_{\mathrm{A}} \geqslant(1+\alpha)\left(\frac{m_{i}}{m_{e}}\right)^{\frac{2}{3}} \simeq 26,
$$

where the last expression is derived for $\alpha=0.2$ and the mass ratio, $m_{i} / m_{e}=100$, used in our simulations.

Our choice of Alfvénic Mach numbers, $M_{\mathrm{A}} \geqslant 30$, should therefore in all cases lead to the formation of shocks with strong electrostatic Buneman waves in the foot region. However, we note that even a moderate variation in the simulation parameters (e.g., $M_{\mathrm{A}}, \beta_{\mathrm{p}}$, magnetic-field configuration) may introduce significantly different results: from the absence of a nonthermal population (Wieland et al. 2016) to a large nonthermal fraction produced by the Buneman instability and adiabatic heating (Matsumoto et al. 2012). Here we investigate the impact of the magnetic-field configuration; namely, the angle between the regular magnetic field and the simulation plane. We expect that these simulations may give us a better understanding of acceleration processes in high-Mach-number shocks under fully 3D geometry.

Early 1D simulations (Quest 1985; Lembege \& Dawson 1987) and recent 2D simulations (Umeda et al. 2008, 2009, 2014; Wieland et al. 2016) indicated that supercritical perpendicular shocks become nonstationary and undergo cyclic self-reformation. Therefore, all instabilities driven by reflected ions in the foot region will also evolve in a nonstationary way, and the conditions for the Buneman-wave growth and efficient SSA will probably be met only at certain locations and time periods. Here we follow the shock evolution long enough (i.e., for eight ion cyclotron times) to demonstrate this influence.

The paper is organized as follows. We present description of simulations setup in Section 2. The results are presented in Section 3. Discussion and summary are in Section 4.

\section{Simulation Setup}

In our simulations, two counter-streaming electron-ion plasma beams of equal density collide with each other to form a system of two shocks propagating in opposite directions that are separated by a contact discontinuity (CD; see Figure 1). The plasma flow is aligned with the $x$-direction, and the streaming velocities of the two slabs are $\boldsymbol{v}_{\mathrm{L}}=v_{\mathrm{L}} \hat{\boldsymbol{x}}$ and $\boldsymbol{v}_{\mathrm{R}}=v_{\mathrm{R}} \hat{\boldsymbol{x}}$, where the indices $\mathrm{L}$ and $\mathrm{R}$ refer, respectively, to the left and right sides of the simulation box, where the beams are injected. The two beams carry a homogeneous magnetic field, $\boldsymbol{B}$, that is perpendicular to the flow direction and lies in the $y z$ plane, forming an angle $\varphi$ with the $y$-axis. As the magnetic field is assumed to be frozen in the moving plasma, a motional electric field $\boldsymbol{E}=-\boldsymbol{v} \times \boldsymbol{B}$ is also initialized in the left and right beam, with $\boldsymbol{v}=\boldsymbol{v}_{\mathrm{L}}$ or $\boldsymbol{v}=\boldsymbol{v}_{\mathrm{R}}$, respectively. The magnetic field strength in both plasmas is equal, $\boldsymbol{B}_{\mathrm{L}}=\boldsymbol{B}_{\mathrm{R}}$, and since $v_{\mathrm{L}}=-\boldsymbol{v}_{\mathrm{R}}$, the motional electric field has opposing signs in the two slabs. To avoid an artificial electromagnetic transient resulting from this strong gradient in the motional electric field when the two plasma beams start to interact, we use a modified flow-flow method of shock excitation, recently developed by us and described in Wieland et al. (2016). This method implements a transition zone between the plasma beams, in which the electromagnetic fields are tapered off until they vanish in a small plasma-free area that initially separates the beams. Stability is provided by a current sheet that compensates $\boldsymbol{\nabla} \times \boldsymbol{B}$ in the transition layer. In addition to providing a clean initialization setup, our method allows one to assume different physical conditions in the colliding beams; e.g., the asymmetry in the density of the slabs, as applied in Niemiec et al. (2012) and Wieland et al. (2016).

Here we assume different temperatures for the left and the right plasma beams, which otherwise have the same physical characteristics, including density. Specifically, we set the plasma beta (the ratio of the plasma pressure to the magnetic pressure) in the left slab to $\beta_{\mathrm{p}, \mathrm{L}}=0.0005$ and in the right slab to $\beta_{\mathrm{p}, \mathrm{R}}=0.5$. The thermal velocities of plasma particles in the two beams thus differ by a factor of $\sqrt{1000} \simeq 30$, which is the difference in the sonic Mach numbers, $M_{\mathrm{S}}$, of the shocks that form on both sides of the CD. Note that our choice of plasma beta $\beta_{\mathrm{p}}=0.5$ for one of the shocks allows for a direct comparison with the results of Matsumoto et al. (2012, 2013).

The counter-streaming plasma beams move with equal absolute velocities, $v_{\mathrm{L}}=0.2 c=v_{\mathrm{R}}$, so that they collide with a relative velocity of $v_{\text {rel }} \simeq 0.38 c$, where $c$ is the speed of light. Our simulation frame is the center-of-momentum frame of the system. Upon plasma collision, two shocks form and propagate away from the CD in the left and the right plasma. Here we refer to these shocks as to the left and the right shock, respectively. Because the two unshocked plasmas are cold, the system remains in approximate ram-pressure balance throughout the simulation, and the $\mathrm{CD}$ is stationary in the simulation frame. Therefore, the simulation frame is coincident with the downstream rest frames of the two shocks. 


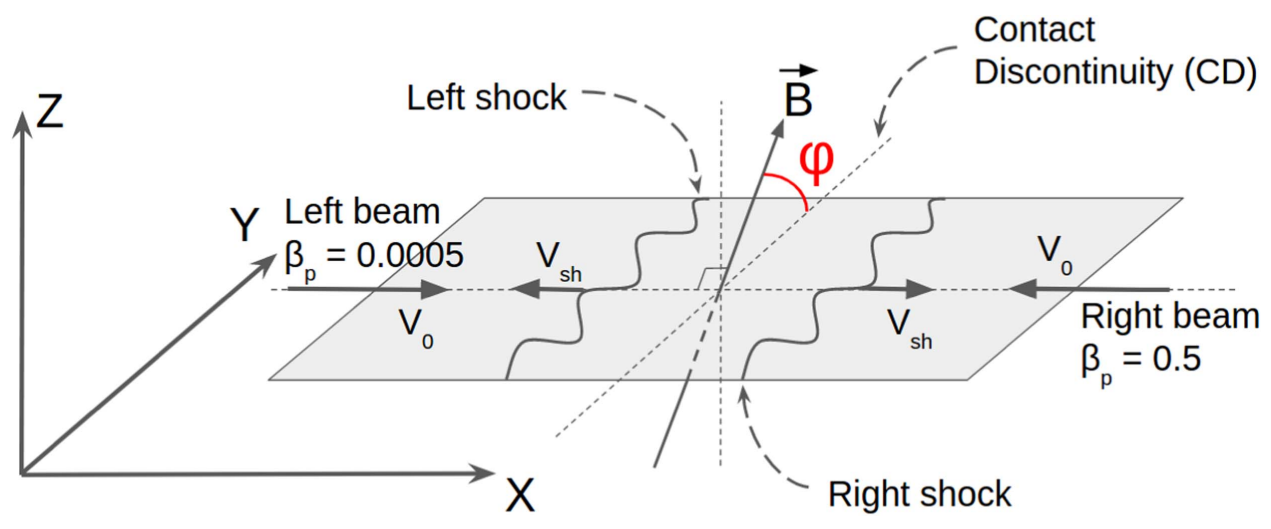

Figure 1. Illustration of the simulation setup.

Restricted by available computational resources, we perform our simulations using a 2D3V model; i.e., we keep track of all three components of particle velocities and electromagnetic fields, and follow particle positions only in the $x y$ plane. Since, as we show, in such a geometry the physics depends on the orientation of the initially uniform perpendicular magnetic field with respect to the simulation plane, we carry out numerical experiments for three values of the angle $\varphi$; namely, in-plane magnetic field $\varphi=0^{\circ}$ (runs A1 and A2), $\varphi=45^{\circ}$ (runs B1 and B2), and out-of-plane magnetic field $\varphi=90^{\circ}$ (runs $\mathrm{C} 1$ and $\mathrm{C} 2$; see Figure 1). Run-specific parameters are listed in Table 1. Note that the digits in the run designations above refer to the left and right shock, respectively, that formed in plasma with different temperatures. They are listed here as separate runs to ease a comparison between the cases of cold $\left(\beta_{\mathrm{p}}=5 \cdot 10^{-4}\right.$; runs $\left.\mathrm{A} 1, \mathrm{~B} 1, \mathrm{C} 1\right)$ and moderate $\left(\beta_{\mathrm{p}}=0.5\right.$; runs $\mathrm{A} 2, \mathrm{~B} 2, \mathrm{C} 2$ ) plasma beta.

As noted, the magnetic field in both plasma beams is initially equal, $\boldsymbol{B}=\boldsymbol{B}_{0}$. We consider a weakly magnetized plasma and the ratio of the electron plasma frequency, $\omega_{\mathrm{pe}}=\sqrt{e^{2} N_{e} / \epsilon_{0} m_{e}}$, to the electron gyrofrequency, $\Omega_{\mathrm{e}}=e B_{0} / m_{e}$, which is fixed to $\omega_{\mathrm{pe}} / \Omega_{\mathrm{e}}=12$. Here, $e$ and $m_{e}$ are the electron charge and mass, $\epsilon_{0}$ is the vacuum permittivity, and $N_{e}$ is the electron density. This value of plasma magnetization has been chosen in order to satisfy the trapping condition of the electron in the Buneman waves excited in the shock foot (see Section 1). We further assume the ion-to-electron mass ratio of $m_{i} / m_{e}=100$ and use 20 particles per cell per particle species for both plasma slabs. With this choice, the Alfvén velocity is numerically $v_{\mathrm{A}}=\left[B_{0}^{2} / \mu_{0}\left(N_{e} m_{e}+N_{i} m_{i}\right)\right]^{1 / 2}=8.29 \times 10^{-3} c$, where $\mu_{0}$ is the vacuum permeability.

The sonic and Alfvénic Mach numbers of the two shocks depend on the orientation angle of the uniform magnetic field with respect to the simulation plane, $\varphi$. For configurations with $\varphi=0^{\circ}$ and $\varphi=45^{\circ}$ (runs A and B), the large-scale field bends particle trajectories out of the simulation plane. Particles thus effectively have three degrees of freedom, hence a nonrelativistic adiabatic index $\Gamma=5 / 3$. For the out-of-plane field configuration $\left(\varphi=90^{\circ}\right.$, run $\left.\mathrm{C}\right)$, particles are tied to the $2 \mathrm{D}$ simulation plane, have two degrees of freedom, and $\Gamma=2$. The resulting sound speeds thus differ, and for low plasma beta $\left(\beta_{\mathrm{p}}=5 \times 10^{-4}\right)$ they $\operatorname{read} c_{\mathrm{S}}=\left(\Gamma k T_{\mathrm{i}} / m_{i}\right)^{1 / 2} \simeq 1.7 \times 10^{-4} c$ in runs $\mathrm{A} 1$ and $\mathrm{B} 1$, and $c_{\mathrm{s}} \simeq 1.86 \times 10^{-4} \mathrm{c}$ in run $\mathrm{C} 1$. For a moderate plasma beta $\left(\beta_{\mathrm{p}}=0.5\right.$; runs A2-C2), the sound speeds are a factor of $\sqrt{1000}$ larger. The compression ratio at the shock also depends on $\Gamma$, and
Table 1

Parameters of the Simulations and Derived Shock Properties

\begin{tabular}{lcccrl}
\hline \hline Run & $\varphi$ & $L_{y}\left(\lambda_{\mathrm{si}}\right)$ & $M_{\mathrm{A}}$ & $M_{\mathrm{s}}$ & \multicolumn{1}{c}{$\beta_{\mathrm{p}}$} \\
\hline A1 & $0^{\circ}$ & 24 & 31.7 & 1550 & 0.0005 \\
$\mathrm{~A} 2$ & $0^{\circ}$ & 24 & 31.7 & 49 & 0.5 \\
\hline B1 & $45^{\circ}$ & 24 & 31.7 & 1550 & 0.0005 \\
B2 & $45^{\circ}$ & 24 & 31.7 & 49 & 0.5 \\
\hline C1 & $90^{\circ}$ & 12 & 35.5 & 1581 & 0.0005 \\
C2 & $90^{\circ}$ & 12 & 35.5 & 55 & 0.5 \\
\hline
\end{tabular}

Note. Parameters of the simulation runs described in this paper. Listed are: the orientation of the uniform perpendicular magnetic field with respect to the $2 \mathrm{D}$ simulation plane, $\varphi$, the transverse size of the computational box size in units of the ion skin depth, $\lambda_{\mathrm{si}}=200 \Delta$, Alfvénic and sonic Mach numbers of the shocks, $M_{\mathrm{A}}$ and $M_{\mathrm{s}}$, and the plasma beta, $\beta_{\mathrm{p}}$, of the upstream plasmas. All runs use an electron skin depth of $\lambda_{\mathrm{se}}=20 \Delta$, the ion-electron mass ratio $m_{i} / m_{e}=100$, and the plasma magnetization $\omega_{\mathrm{pe}} / \Omega_{\mathrm{e}}=12$.

is $r=3.97$ and $r=2.98$ for runs $\mathrm{A}-\mathrm{B}$ and $\mathrm{C}$, respectively. The expected shock speeds in the simulation frame are $v_{\mathrm{sh}} \simeq 0.067 c$ for runs $\mathrm{A}$ and $\mathrm{B}$, and $v_{\mathrm{sh}} \simeq 0.1 c$ for run $\mathrm{C}$. The shock speeds in the upstream frame are $0.263 c$ and $0.294 c$, respectively. We calculate the sonic and Alfvénic Mach numbers of the shocks in the upstream reference frame, and their values are provided in Table 1. Note that for the parameters assumed in our simulations the shocks easily satisfy both the unstable $\left(M_{\mathrm{s}} \geqslant 6\right)$ and the trapping condition $\left(M_{\mathrm{A}} \gtrsim 25.8\right)$, where our estimate uses $\alpha=0.2$ in Equations (1) and (2). Thus, in all cases we should expect efficient electron acceleration.

The electron skin depth in the upstream plasma is common in all simulation runs and equals $\lambda_{\mathrm{se}}=c / \omega_{\mathrm{pe}}=20 \Delta$, where $\Delta$ is the size of the grid cells. For the assumed mass ratio, the ion skin depth is $\lambda_{\mathrm{si}}=200 \Delta$. Here we use $\lambda_{\mathrm{si}}$ as the unit of length. The timescale and all of the temporal dependencies are given in terms of the upstream ion Larmor frequency $\Omega_{\mathrm{i}}^{-1}=120 \omega_{\mathrm{pi}}^{-1}=$ $1200 \omega_{\mathrm{pe}}^{-1}=100 \Omega_{\mathrm{e}}^{-1}$, where $\Omega_{\mathrm{i}}=e B_{0} / m_{i}$. The simulation time, $t=8 \Omega_{i}^{-1}$, is chosen to cover at least three shock reformation cycles. The time-step we use is $\delta t=1 / 40 \omega_{\mathrm{pe}}^{-1}=1 / 48000 \Omega_{\mathrm{i}}^{-1}$.

The two plasma beams are composed of an equal number of ions and electrons, initialized at the same locations to ensure the initial charge-neutrality. Plasma is continuously injected at both sides of the simulation box. The injection layer moves away from the interaction region and is at all times kept at a 
sufficient distance in order to contain all of the reflected particles and generated electromagnetic fields in the computational box. At the same time, the distance is close enough so that the beam does not travel too long without any interaction, which suppresses numerical grid-Cerenkov effects and saves computational resources. The simulation box thus expands during an experiment in the $x$-direction, and can reach a final size $L_{x}=250 \lambda_{\mathrm{si}}=50,000 \Delta$ at the end of the run. Open boundary conditions are imposed in the $x$-direction.

The transverse size of the simulation box is $L_{y}=$ $24 \lambda_{\mathrm{si}}=4800 \Delta$ for runs $\mathrm{A}$ and $\mathrm{B}$, and $12 \lambda_{\mathrm{si}}$ for run $\mathrm{C}$. Periodic boundaries are applied in the $y$-direction. The box size for runs $\mathrm{A}$ and $\mathrm{B}$ is larger because in these cases we expect to observe turbulent magnetic reconnection within the shock structure (Matsumoto et al. 2015), the proper investigation of which requires appropriate statistics for magnetic filaments formed in the shock ramp. The transverse box size is significantly larger than those used in earlier studies (e.g., Matsumoto et al. 2012, 2015; Wieland et al. 2016).

The code used in this study is a 2D3V-adapted and modified version of the relativistic electromagnetic particle code TRISTAN with Message Passing Interface-based parallelization (Buneman 1993; Niemiec et al. 2008). The numerical model is essentially the same as that used in Wieland et al. (2016). A notable addition is the possibility of following individual selected particle trajectories, which allows us to study particle acceleration processes in detail. Convergence studies with various PIC numbers, $N_{\mathrm{ppc}}=10-40$, and the values of the reduced mass ratio, $m_{i} / m_{e}=50-400$, have been performed in order to verify that the essential physical processes are correctly reproduced for the parameters used here.

\section{Results}

In what follows, we present a comparison of three types of simulations that differ in the orientation of the upstream magnetic field: in-plane $\left(\phi=0^{\circ}\right.$, runs A1 and A2), $\phi=45^{\circ}$ (runs B1 and B2), and out-of-plane ( $\phi=90^{\circ}$, runs $\mathrm{C} 1$ and $\mathrm{C} 2$ ). We discuss the overall shock structure, differences in the evolution of the Buneman modes, the influence of these modes on electron pre-acceleration, the main features of electron acceleration for the three magnetic-field configurations, the resulting electron spectra downstream of the shock, and the influence of shock self-reformation on all these processes.

\subsection{Global Shock Structure}

In this section we give an overview of the shock structures observed for the three cases in this study. We base our description on the left shocks propagating into cold plasma (i.e., runs A1-C1), and discuss results for shocks developing in a moderate-temperature plasma (i.e., runs A2-C2) only where they differ from those for $\beta_{\mathrm{p}} \ll 1$.

Figures 2 and 3 show electron density maps and the ion phase-space in the shock region in panels (a)-(c), respectively, for runs A1, B1, and C1. They present the system at times close to the end of the simulation runs, specifically at a phase of shock reformation when the largest number of shock-reflected ions appear and the Buneman waves in the shock foot reach maximum amplitudes. These times differ slightly between the runs, and are $t \Omega_{\mathrm{i}}=7.625$ for run $\mathrm{A} 1, t \Omega_{\mathrm{i}}=7.5$ for run $\mathrm{B} 1$,
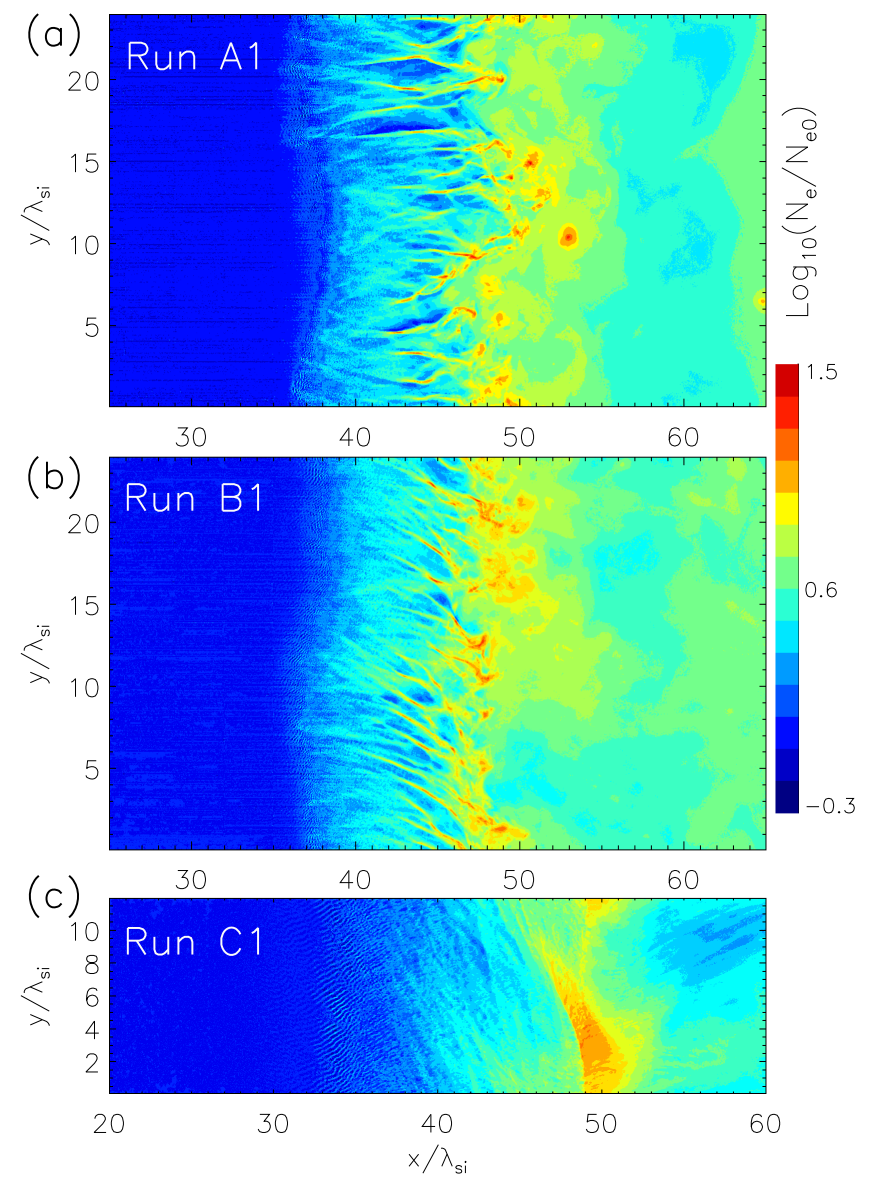

Figure 2. Distributions of the normalized electron number density at shocks propagating in cold plasmas. Panel (a): run $\mathrm{A} 1$ at $t \Omega_{\mathrm{i}}=7.625$, panel (b): run $\mathrm{B} 1$ at $t \Omega_{\mathrm{i}}=7.5$, and panel (c): run $\mathrm{C} 1$ at $t \Omega_{i}=7.75$. A logarithmic scaling is used.

and $t \Omega_{i}=7.75$ for run $\mathrm{C} 1$. Note that the system already contains fully formed self-sustained shocks.

As expected for supercritical shocks, their structures are determined by the fraction of upstream plasma ions that are reflected from the shock front. In our case of perpendicular shocks, the reflection is due to the shock-compressed magnetic field. Reflected ions gyrate around the magnetic-field lines in the upstream region, exciting various plasma instabilities. For shocks with high Alfvén Mach numbers, the most important instabilities are the Weibel-type filamentation instability in the shock ramp and the Buneman instability in the shock foot (Wieland et al. 2016). Ion reflection also leads to the so-called overshoot; i.e., plasma compression at the shock front that exceeds the compression expected from the Rankine-Hugoniot conditions in the MHD description. The overshoot in run $\mathrm{C} 1$ with the out-of-plane uniform magnetic field can be approximately identified with a largely coherent compression structure at $x / \lambda_{\mathrm{si}} \approx 50$ in Figure 2(c) (compare also Figure 3 ). In cases $\mathrm{A} 1$ and $\mathrm{B} 1$, the shock transition does not produce a coherent structure, but in the density profiles averaged over the $y$-direction (not shown) the overshoot is located at $x / \lambda_{\mathrm{si}} \approx 50$ and $x / \lambda_{\mathrm{si}} \approx 48$, respectively, for runs $\mathrm{A} 1$ and B1. Downstream of the overshoot the plasma density oscillates around an average value that is commensurate with that expected in the MHD picture (see Sections 1 and 3.4.4).

The Weibel-type filamentation instability results from the interaction between shock-reflected and incoming plasma ions. 


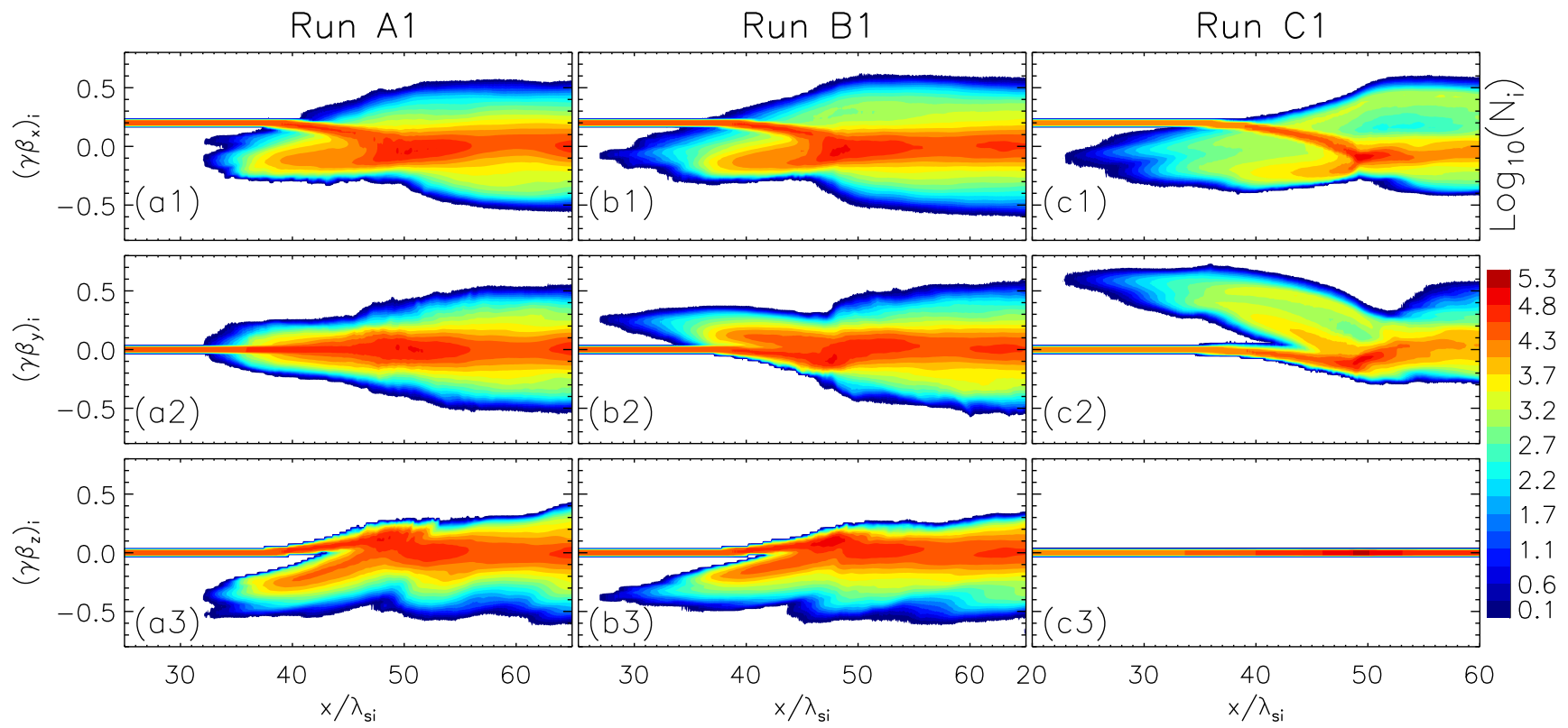

Figure 3. Phase-space distributions of ions for the left shock regions shown in Figure 2, averaged over the spatial coordinate $y$. From top to bottom, shown are the $x, y$, and $z$-components of particle momenta, $\gamma \beta$, from left to right, for run $\mathrm{A} 1, \mathrm{~B} 1$, and $\mathrm{C} 1$. A logarithmic scaling is used.

It leads to the formation of magnetic filaments with a separation scale that is of the order of the ion skin depth, $\lambda_{\mathrm{si}}$. The filaments can be identified in the density distributions (Figure 2) in the shock-ramp region between the overshoot and the shock foot; i.e., for $x / \lambda_{\mathrm{si}} \approx(38-50),(38-48)$, and (36-50), for runs $\mathrm{A} 1$, $\mathrm{B} 1$, and $\mathrm{C} 1$, respectively. The structure and geometry of filaments depend on the configuration of the uniform magnetic field because it defines the gyration direction of the reflected ions. Figure 3 demonstrates that in the case of an in-plane magnetic field $\left(\varphi=0^{\circ}\right.$, Figure 3(a)), ions are reflected primarily in the $x z$ plane, which leads to the formation of the density filaments along the plasma flow direction that we see in Figure 2(a). For the configuration with $\varphi=45^{\circ}$ (Figure 3(b)), in addition to negative $v_{x}$ and $v_{z}$ components of the reflected ion velocity, there is a positive $v_{y}$ velocity component that causes the density filaments to become oblique. Finally, for the out-of-plane configuration $\left(\varphi=90^{\circ}\right.$, Figure 3(c)), the reflected ions are confined to the simulation plane, again resulting in density filaments that are blurred in Figure 2 on account of their large obliquity.

The waves visible in the density distributions in Figure 2 upstream of the shock ramp, in the shock foot regions, $\left(x / \lambda_{\mathrm{si}} \approx(34-38)\right.$ for runs $\mathrm{A} 1$ and $\mathrm{B} 1$, and $x / \lambda_{\mathrm{si}} \approx(30-36)$, for run $\mathrm{C} 1$ ), have a different nature and result from the electrostatic Buneman instability caused by the interaction between the shock-reflected ions and inflowing upstream electrons. Their wavelength is much smaller than the ion inertia length, and the wave vector is approximately orthogonal to that of the magnetic filaments. The Buneman waves are discussed in detail in Section 3.3.

It was demonstrated by Matsumoto et al. (2015) that for the in-plane magnetic field configuration, merging magnetic filaments in the shock ramp can trigger spontaneous turbulent magnetic reconnection, providing an additional channel for electron acceleration. We observe magnetic reconnection for both the in-plane and $\varphi=45^{\circ}$ magnetic field configurations (runs A and B for the cold and moderate-temperature plasmas). The magnetic-reconnection events can be identified by chains

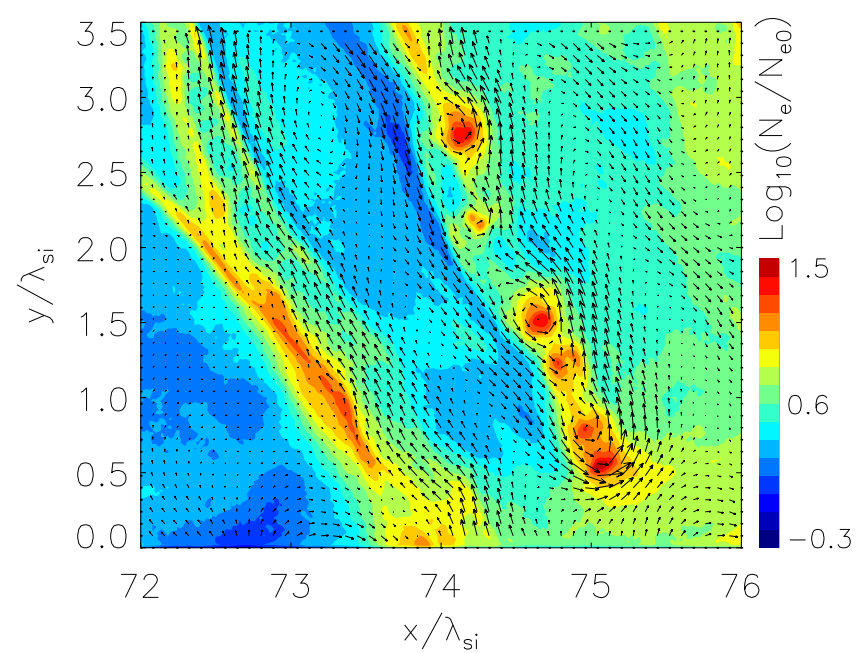

Figure 4. Blow-up of the region containing a chain of magnetic islands resulting from magnetic reconnection in the filamentary structures of the shock ramp. The snapshot was taken for run $\mathrm{B} 1$ at time $t=3.8 \Omega^{-1}$. The normalized density is color-coded in logarithmic scale. Arrows show the in-plane $(x-y)$ component of the magnetic field.

of magnetic islands separated by X-points, which result from the nonlinear decay of the current sheets (Furth et al. 1963) Corresponding enhancements lined up along magnetic filaments are visible in plasma density, an example of which is shown in Figure 4. The role of the magnetic-reconnection specific acceleration processes will be discussed in detail in a follow-up paper.

\subsection{Cyclic Shock Reformation}

Our previous investigation of perpendicular shocks in the regime of high Alfvén Mach numbers (Wieland et al. 2016) demonstrated a cyclic shock self-reformation known from earlier studies of low-Mach-number shocks. The process is caused by nonstationary ion reflection off of the shock ramp that at high Mach numbers was shown to be governed by the 


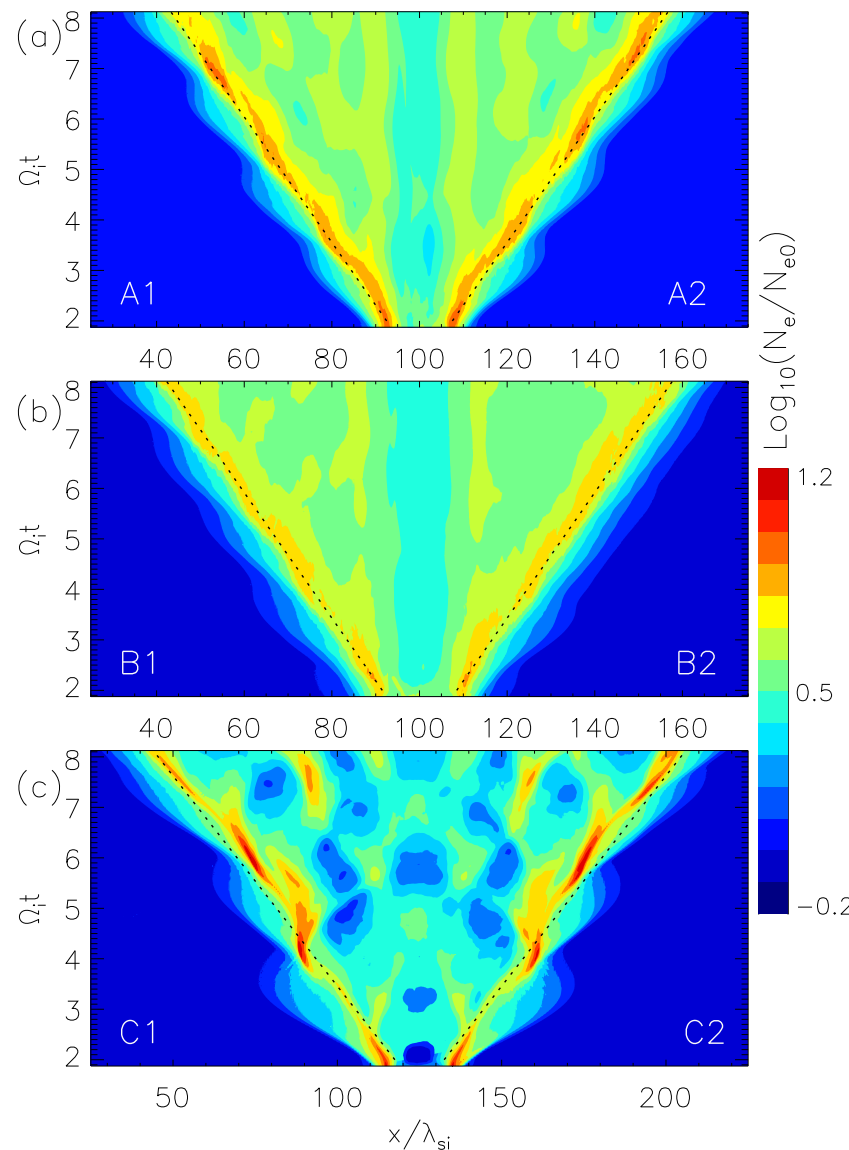

Figure 5. Time development of the electron number density averaged over the $y$-direction for runs A1, A2 (a), runs B1, B2 (b), and runs C1, C2 (c). Color contours present normalized electron density in logarithmic scale. The dashed lines indicate the theoretical shock speed in the simulation frame.

dynamics of magnetic filaments formed by the Weibel-type instability. The timescale of shock reformation is on the order of the gyroperiod of upstream ions. During reformation the shock velocity and plasma density at the shock are quasicyclicly modulated around their average values, and the extension of the filamentary region in the shock ramp varies. A bunch of shock-reflected ions streaming against incoming plasma leads to the formation of current filaments extended along the plasma flow direction that are accompanied in the shock foot by electrostatic Buneman modes. Later in the cycle, once the current filaments start to merge ahead of the shock ramp and become aligned closer to the shock surface on account of bunched ion gyration, the turbulent shock precursor shrinks, and the Buneman modes disappear. This has profound consequences for particle acceleration, as will be discussed in Section 3.4.4 below.

Figure 5 shows for all runs the time evolution of the electron density averaged over the $y$-direction. The motion of the left and right shocks, with an average speed $v_{\mathrm{sh}, \mathrm{L}}$ or $v_{\mathrm{sh}, \mathrm{R}}$, are marked with dashed lines. A self-reformation cycle begins at $T \Omega_{i} \approx 2$, after the shocks have been fully formed. The reformation processes are observed for all magnetic-field orientations and plasma temperatures studied, and are most pronounced for out-of-plane (run C) and in-plane (run A) field configurations. The period of self-reformation varies around the average value $1.55 \Omega_{i}^{-1}$ and is consistent with our earlier finding of approximately $1.5 \Omega_{i}^{-1}$ obtained for the $\varphi=45^{\circ}$ configuration
(Wieland et al. 2016). Rippling modes caused by the spatial modulation of ion reflection (e.g., Burgess \& Scholer 2007; Wieland et al. 2016) are not captured in our simulations because their wavelength is not smaller than the transverse size of the simulation box.

The velocities of the left and the right shock in the simulation (or downstream) reference frame (calculated as an overshoot speed) vary between $0.03 c$ and $0.15 c$. The average shock speed equals $0.066 c$ for runs $\mathrm{A} 1$ and $\mathrm{A} 2,0.067 c$ for runs $\mathrm{B} 1$ and $\mathrm{B} 2$, and $0.094 c$ for runs $\mathrm{C} 1$ and $\mathrm{C} 2$, which is very close to the expected speeds of $0.066 c$ and $0.1 c$, respectively.

\subsection{The Buneman Instability}

As discussed above, the Buneman instability results from the interaction of shock-reflected ions with the upstream electrons. It has been shown that this instability can be excited in the foot of high-Mach-number nonrelativistic perpendicular shocks. The properties of these electrostatic modes depend on physical parameters such as the shock Mach number and plasma temperature. In addition, the instability characteristics may be different in numerical studies with restricted dimensionality. In particular, in 2D simulations the orientation of the average magnetic field with respect to the simulation plane may play a role, as suggested from a comparison of studies applying the out-of-plane magnetic field (e.g., Matsumoto et al. 2012, 2013), the $\varphi=45^{\circ}$ configuration (e.g., Wieland et al. 2016), and the in-plane field (e.g., Kato \& Takabe 2010). However, all of these works differ in the specific physical parameters assumed. In contrast, our present study enables a direct comparison of the Buneman instability properties in simulation runs in which the main variable parameter is the magnetic field orientation.

Figure 6 presents a blow-up of a portion of the left shock foot shown in Figures 2 and 3. From top to bottom, displayed are the electron density, the $E_{x}$ component of the electric field, and the electrostatic field amplitude, from left to right for runs A1, $\mathrm{B} 1$, and $\mathrm{C} 1$. The electrostatic field amplitude is calculated as $\left|E_{\text {est }}\right|=|-\nabla \phi|$, where $\phi$ is the electric potential derived directly from the charge distribution. The modes in the electrostatic field maps in Figures 6(a3)-(c3) appear to have half their true wavelength, because the absolute values of $\nabla \phi$ are plotted and one cannot distinguish between positive and negative field strengths. The wave structures visible in the plot occur at scales much smaller than the ion inertia scale. The form of the waves depends on the magnetic-field configuration. For the out-of-plane field orientation (run C1, Figure 6(c)), coherent wave trains are formed over an extended region of the shock foot (see, e.g., Matsumoto et al. 2012). The electrostatic field reaches amplitudes of $\left|E_{\mathrm{est}}\right| / B_{0} \sim 2$, and the trapping condition is easily satisfied. Wave coherence is broken for the in-plane configuration (run A1, Figure 6(a)), and the modes are distributed in patchy structures formed in narrow regions ahead of the magnetic filaments (see Figure 6(a1)). In the $\varphi=45^{\circ}$ case, the appearance is intermediate between these forms-the wave structure is largely coherent but over a narrow region with thickness $\sim \lambda_{\mathrm{si}}$. For both $\mathrm{A} 1$ and B1, the amplitude of the electrostatic field reach $\left|E_{\text {est }}\right| / B_{0} \sim 1.7$ at peak locations, but the average field strength is $\left|E_{\text {est }}\right| / B_{0} \sim 1$ or less.

A significant part of the variation in the coherence and the volume coverage between runs $\mathrm{A} / \mathrm{B}$ and $\mathrm{C}$ arises from the homogeneity of the reflected ion beam which in turn depends on the structure of the overshoot. Ion reflection in the out-ofplane case is largely uniform along the shock because the shock 

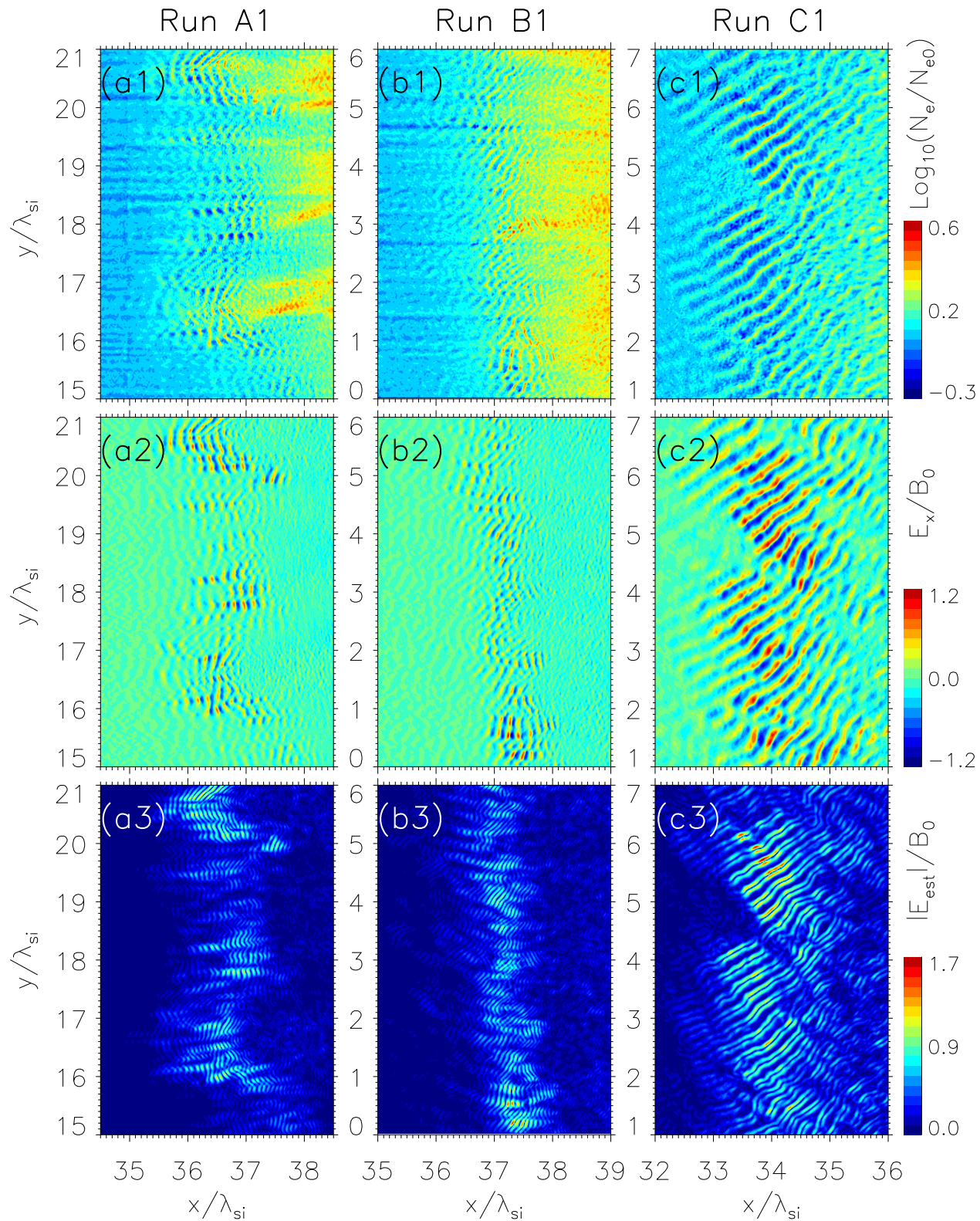

Figure 6. Electron density (a1-c1), the $x$ component of the electric field (a2-c2), and electrostatic field amplitudes (a3-c3) in selected regions of the shock foot with the most intense Buneman waves for runs A1 ( $\mathrm{a}^{*}$ panels), B1 ( $\left.\mathrm{b}^{*}\right)$, and $\mathrm{C} 1\left(\mathrm{c}^{*}\right)$. Density is presented in logarithmic scaling and normalized to the upstream density. Electric and electrostatic field strengths are normalized to that of the upstream magnetic field.

overshoot has a fairly coherent structure. The overshoot region for $0^{\circ}$ and $45^{\circ}$ magnetic-field orientations has clumpy structures producing an incoherent flow of reflected ions.

As noted in Section 3.2, the intensity of the electrostatic waves varies considerably during a shock reformation cycle on account of the changing number of shock-reflected ions. The average value of the electrostatic field amplitude varies in the range $\left|E_{\text {est }}\right| / B_{0} \sim 0.1-1.1$ for run $\mathrm{A} 1,0.3-1.1$ for run $\mathrm{B} 1$, and 0.3-1.5 for run $C 1$. Note also that for the right shocks in moderate-temperature plasma, $\beta_{\mathrm{p}}=0.5$, the maximum intensity of the Buneman waves is $20 \%-50 \%$ smaller than in the cold plasma. However, the area of the unstable region is $20 \%-30 \%$ larger in the case of warm plasma. We do not expect a thermal reduction in the Buneman growth rate that would explain the lower wave intensity, and we cannot exclude the fact that the saturation level is lower on account of strong nonlinear Landau damping (Brě̌zman \& Ryutov 1974) or the modulation instability (Galeev et al. 1977). The efficiency of electron acceleration is determined by the number of trapped electrons, and the volume fraction occupied by intense Buneman modes with the convective electric field that provides the acceleration. A conjunction of these factors, though different in each case analyzed here, permit efficient electron acceleration at both the low- and moderate- $\beta_{\mathrm{p}}$ shocks.

The Buneman waves observed in the shock foot have a wave vector

$$
k=\frac{c}{\Delta v} \frac{1}{\lambda_{\mathrm{se}}},
$$

where $\Delta v$ is the relative speed between incoming electrons and reflected ions, and they are excited when $\Delta v$ exceeds the thermal speed of the electrons, $\Delta v / v_{\text {th }} \gg 1$, an expression that is equivalent to that given in Equation (1). The beam of 


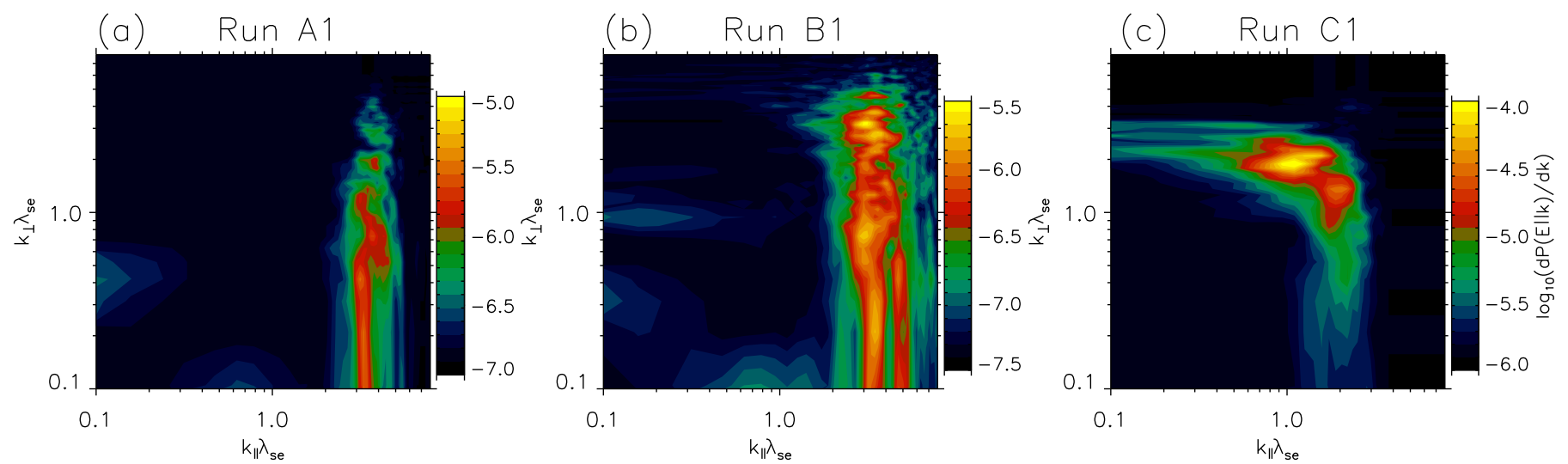

Figure 7. Fourier power spectrum of the electric field parallel to the wave vector, $\boldsymbol{e}_{k} \cdot \boldsymbol{E}$, for the regions presented in the panels a1 (a), b1 (b), and c1 (c) of Figure 6.

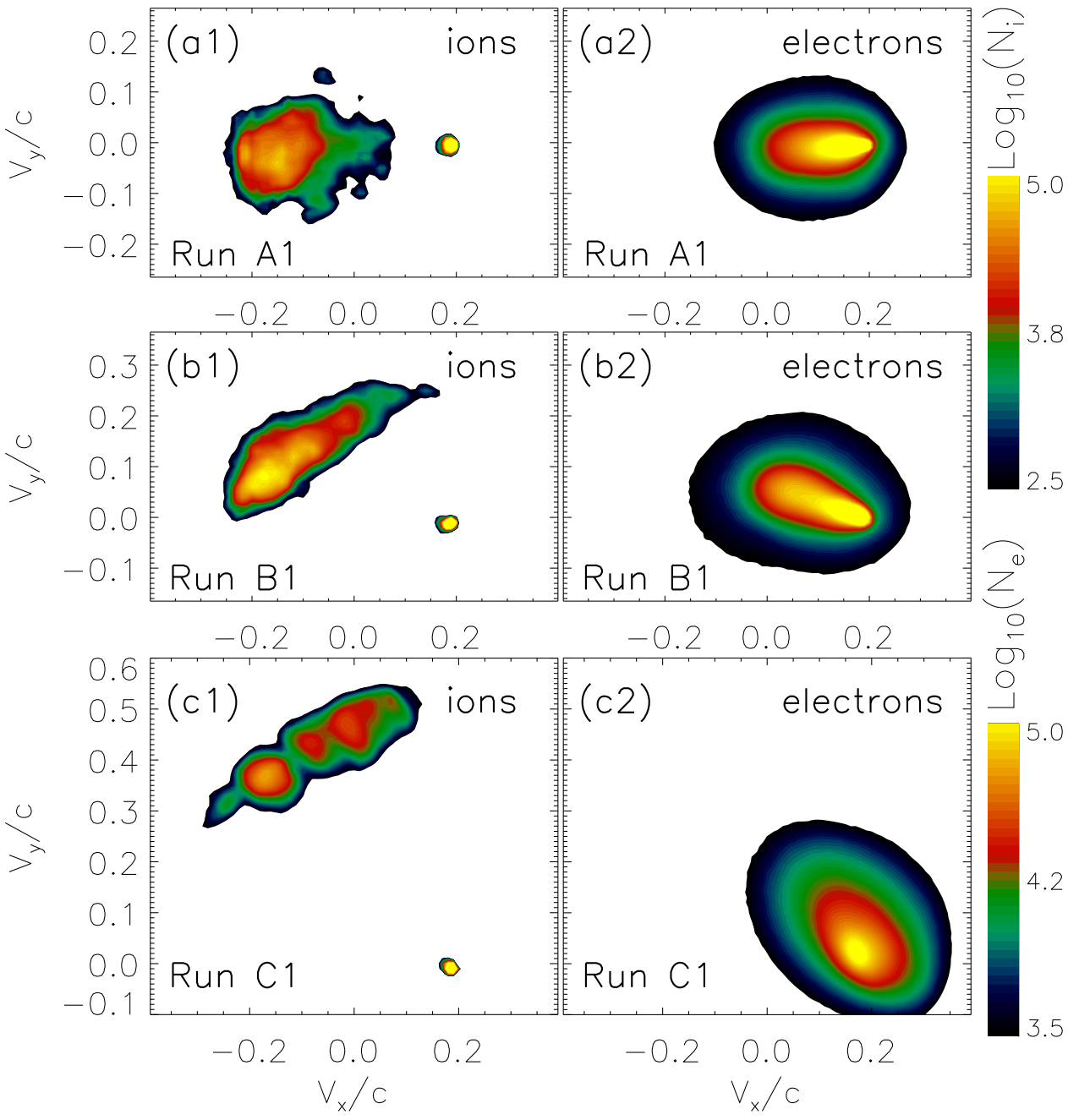

Figure 8. Phase-space distribution in $v_{x}-v_{y}$ of ions (first column) and electrons (second column) in the regions presented in Figure 6 for runs A1 (a1, a2), B1 (b1, b2), and $\mathrm{C} 1$ (c1, c2). The scale is logarithmic.

reflected ions is warm, and we expect the electrostatic instability to operate in the kinetic regime and the wave vector of the peak intensity to be aligned with the streaming velocity (Breizman 1990). Figure 7 displays Fourier power spectra of the electric field parallel to the wave vector, $\boldsymbol{e}_{k} \cdot \boldsymbol{E}$, calculated in the regions shown in Figure 6. The mean field values and linear gradients were removed from the $E_{x}$ and $E_{y}$ maps for this analysis. Corresponding ion and electron phase-space distributions in $v_{x}-v_{y}$ are shown in Figure 8. For run A1 we see a narrow signal at $k_{\|} \lambda_{\text {se }} \simeq 3.3$ in the range $k_{\perp} \lambda_{\text {se }} \lesssim 0.7$ (Figure 7(a)) that corresponds to $k \simeq 3.3 \lambda_{\mathrm{se}}^{-1}$ and $\Delta v \simeq 0.3 c$. Noting that the plasma motion in this case (A) is primarily along the $x$-direction and calculating averaged velocity components, we obtain $v_{\mathrm{xi}} \simeq-0.15 c$ and $v_{\mathrm{xe}} \simeq 0.17 c$ for reflected ions and incoming electrons, respectively (compare Figure 8(a)). The relative velocity is thus $\Delta v \simeq 0.31 c$, in good 
agreement with the value calculated from the Fourier spectrum. The modes can be clearly identified with the Buneman waves.

For the simulation with $\varphi=45^{\circ}$ (run B1) we see the mode out to $k_{\perp} \lambda_{\text {se }} \simeq 4$. The phase-space plot (Figure $8(\mathrm{~b})$ ) suggests that the parallel mode should be observed between $\boldsymbol{k} \lambda_{\mathrm{se}} \simeq(3 ; 0)$ and $\boldsymbol{k} \lambda_{\mathrm{se}} \simeq(2.5 ; 3)$, which is again in rough agreement with the range of wave vectors for which the Fourier analysis indicates a high intensity of $\boldsymbol{e}_{k} \cdot \boldsymbol{E}$ wave modes.

For the out-of-plane magnetic field configuration the velocity range of reflected ions inserted in Equation (3) implies strong growth between $\boldsymbol{k} \lambda_{\text {se }} \simeq(1.4 ; 1.4)$ and $\boldsymbol{k} \lambda_{\text {se }} \simeq(0.75 ; 2)$, exactly where we observed it in the Fourier spectra.

The Fourier power spectra also indicate wave intensity at wave vectors not aligned with the streaming velocity of reflected ions. Figure $6(\mathrm{c} 2)$ suggests that at least part of that arises from localized reorientation of the wave fronts in $E_{x}$, probably caused by modulation through large-scale modes.

The high electrostatic field amplitudes in run $\mathrm{C}$ are somewhat surprising, because there are fewer reflected ions in this run compared to the simulations with $\varphi=0^{\circ}$ and $\varphi=45^{\circ}$. Amano \& Hoshino (2009b) modified an estimate by Ishihara et al. (1980) for the transferable energy density,

$$
\epsilon_{0} \frac{E^{2}}{2} \simeq \frac{3 N_{e} m_{e} \Delta V^{2}}{8}\left(\frac{m_{e}}{m_{i}}\right)^{1 / 3},
$$

and we find fairly good agreement with the energy density seen in our simulation $(\sim 70 \%)$, provided that we use the component of $\Delta V$ that lies in the simulation plane (and is indicated in Figure 8). Only for run $\mathrm{C}$ (or $\varphi=90^{\circ}$ ) do we find the trajectories of reflected ions fully contained in the simulation plane; therefore, part of the streaming motion in runs A and B cannot excite the Buneman instability because $\boldsymbol{k}$ must lie in the simulation plane and $\boldsymbol{k} \| \boldsymbol{\Delta} \boldsymbol{V}$.

We conclude that the out-of-plane configuration is best suited to fully capturing the development of the Buneman waves in a $2 \mathrm{D} 3 \mathrm{~V}$ simulation, although the adiabatic index is modified in that case. Equation (2) applies in that case, and the velocity difference between the electrons and the reflected ions is $\Delta V \simeq 2^{-0.5} \Delta V(1,1,0)$. If the large-scale magnetic field is not strictly out-of-plane, but stands at an angle $\varphi<90^{\circ}$ to the simulation plane, then the relative motion is partially rotated out of the simulation plane, and

$$
\Delta \boldsymbol{V} \simeq 2^{-0.5} \Delta V(1, \sin \varphi, \cos \varphi) .
$$

The z-component cannot drive Buneman waves, and so we need a larger shock speed to drive the wave intensity in the simulation plane to a level that permits electron trapping. We therefore suggest that formula (2) for the trapping condition be modified to

$$
M_{\mathrm{A}} \geqslant \sqrt{\frac{2}{1+\sin ^{2} \varphi}}(1+\alpha)\left(\frac{m_{i}}{m_{e}}\right)^{\frac{2}{3}} .
$$

After inserting numbers, including the reflection rates measured in the simulation, we find that in run $\mathrm{A}$ we would need $M_{\mathrm{A}} \simeq 39$, and $M_{\mathrm{A}} \simeq 35$ in run $\mathrm{B}$, for efficient trapping. Both simulations are set up with $M_{\mathrm{A}}=31.7$, and so the energy of streaming in the simulation plane seems to be insufficient for driving very strong Buneman modes and permitting shock surfing acceleration. Likewise, condition (6) would be strongly violated in the $\varphi=0^{\circ}$ simulations presented by Matsumoto et al. (2015), and indeed no significant SSA was reported.

Applying a similar modification to the driving condition (Equation (1)) leads to the expression

$$
M_{s} \gtrsim \frac{1+\alpha}{\sqrt{2\left(1+\sin ^{2} \varphi\right)}} \sqrt{\frac{m_{i}}{m_{e}}} \sqrt{\frac{T_{e}}{T_{i}}} .
$$

We also note that the restriction of Buneman waves with a $k_{z}$ component to the $x y$ plane changes the orientation of the potential wells in which electrons can be trapped. SSA arises from motional electric field that accelerates along the electrostatic equipotential surface of the waves, and a geometrical restriction of $\boldsymbol{k}$ will also modify the relevant component of the motional electric field, $\boldsymbol{E} \perp \boldsymbol{k}$. The dominant effect in our simulations, however, appears to be the reduction in the saturation amplitude.

The properties of the Buneman instability region in the foot of the right shock propagating into the warm plasma are similar to those described above for the small- $\beta_{\mathrm{p}}$ left shocks. In both cases, the shock reformation imposes cyclic changes to the appearance of the electric-field fluctuations in the foot region, but some general features of Buneman-wave fields can be summarized as follows.

1. The Buneman modes evolve kinetically and are largely, but not perfectly, parallel to the streaming velocity of reflected ions.

2. The streaming direction of reflected ions depends on the orientation of the large-scale magnetic field in 2D simulations, and so the Buneman modes are approximately parallel to the shock normal for the in-plane magnetic field (run A), turn oblique for $\varphi=45^{\circ}$ (run B), and are highly oblique for the out-of-plane magnetic-field configuration (run $\mathrm{C}$ ).

3. In all six runs the wavelengths of Buneman waves are commensurate with the streaming speed of ions, and hence they are smaller for runs A-B in comparison with run $\mathrm{C}$.

4. The region with high-intensity coherent waves is larger and considerably less patchy for the out-of-plane configuration (run $\mathrm{C}$ ) than it is for an inclined or inplane magnetic field (runs A and B), irrespective of the plasma $\beta_{\mathrm{p}}$.

5. In moderate-temperature plasma (runs ${ }^{*} 2$ ), the peak intensity of Buneman waves is less than that in the cold plasma (runs ${ }^{*} 1$ ), but the surface area occupied by the waves is larger.

\subsection{Electron Acceleration}

The parameters in our simulations should provide suitable conditions for electron acceleration up to nonthermal energies. As is known from earlier studies of high-Mach-number shocks, the electron injection at such shocks is at least a two-stage process that starts with the SSA in the shock foot and then is followed by additional particle energization processes in the shock ramp and around the overshoot. In this section we describe the electron pre-acceleration at a perpendicular shock. Our 2D numerical experiments with various configurations of the average magnetic field with respect to the simulation plane allow us to observe the injection processes from different perspectives. This in turn enables us to draw conclusions on the 


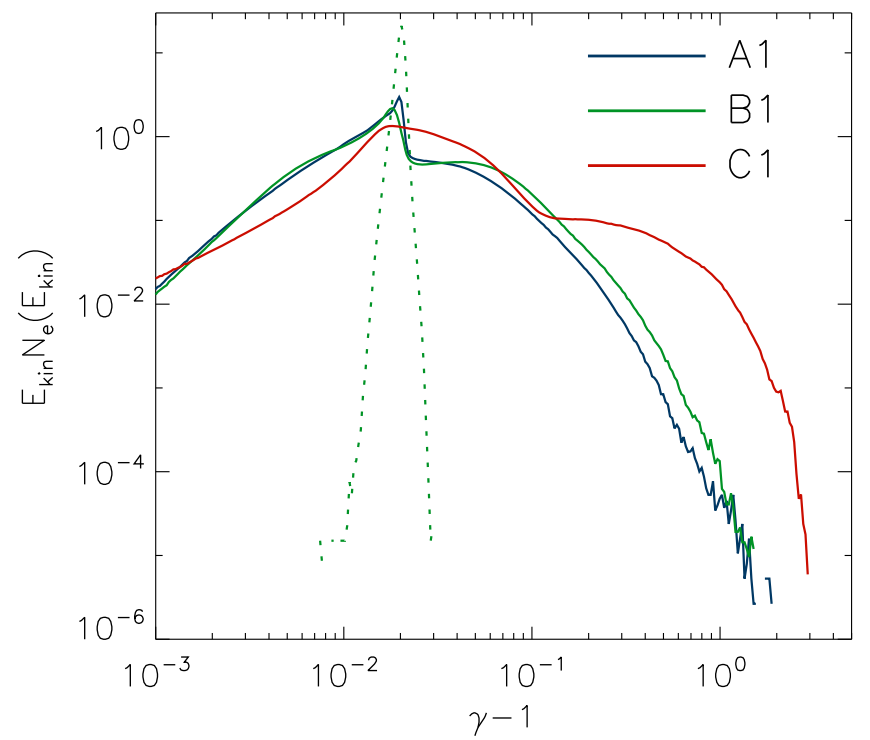

Figure 9. Kinetic energy spectra of electrons in the region harboring Buneman waves as presented in Figure 6 for run A1 (blue), run B1 (green), and run C1 (red). The dotted green line indicates the spectrum of upstream cold plasma electrons (extracted from run B1).

nature of electron pre-acceleration and its true efficiency in a fully 3D system.

We start our discussion in Section 3.4.1 with a description of the SSA process in the shock foot containing the electrostatic Buneman waves. The analysis is based on results of the (left) shocks propagating in cold plasmas with very low plasma beta (runs A1-C1). As noted in Section 3, these shocks provide conditions for strongly nonlinear Buneman modes in the shock foot. Subsequent processes of further electron energization at the shock front are described for shocks in plasmas with a moderate plasma beta (runs A2-C2), at which the injection is more efficient than in the cold plasmas, as we show. The analysis here is presented only for the case of the out-of-plane (run C2, Section 3.4.2) and the in-plane (run A2, Section 3.4.3) magnetic-field configurations, since electron acceleration processes observed in the $\varphi=45^{\circ}$ case are essentially the same as in run $\mathrm{A}\left(\varphi=0^{\circ}\right)$.

\subsubsection{Shock-surfing Acceleration (SSA)}

Figure 9 shows kinetic-energy spectra of electrons residing in the shock-foot regions depicted in Figure 6. For all of the magnetic-field configurations, suprathermal spectral tails are produced by SSA. However, the high-energy portion of the electron spectrum for run $\mathrm{C} 1$ contains a significantly larger number of particles than that found for runs A1 and B1. Specifically, there are about 2200, 7600, and $3.4 \times 10^{6}$ electrons with $\gamma>1.5$ for runs A1, B1, and $\mathrm{C} 1$, respectively. These spectral differences can be explained by the amplitude and filling factor of the Buneman waves in the shock foot, as discussed in Section 3.3. In runs A1 and B1, the wave zone is narrow, and the wave amplitude is barely sufficient for trapping relativistic electrons. On the other hand, in run $\mathrm{C} 1$, the Buneman waves occupy a region that is almost three times larger, and are more intense and coherent. We find that many electrons approaching the foot from the far upstream can interact with electrostatic waves twice or even three times before being advected toward the shock. This multiple SSA processes enable the particles to achieve energies up to $\gamma \sim 4$

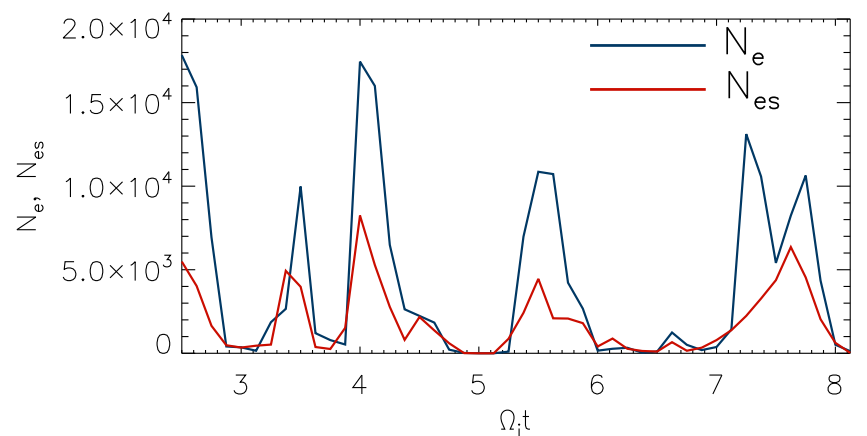

Figure 10. Temporal profiles of the number of energetic electrons, $N_{e}(\gamma>1.5)$ (blue line), compared to the abundance of strong electrostatic field, $N_{\mathrm{es}}\left(E_{\mathrm{es}}>0.5 B_{0}\right)$ (red line). The Spearman rank correlation coefficient is 0.9 , with a $p$-value of $4 \cdot 10^{-17}$. The figure applies to the region harboring Buneman waves in run $\mathrm{A} 1$.

(Amano \& Hoshino 2009a; Matsumoto et al. 2012) before they are further energized in the shock ramp.

There is a clear correlation between the number of preaccelerated electrons and the occurrence of intense electrostatic field. Figure 10 displays for run A1 the temporal development of the number of electrons with a Lorentz factor $\gamma>1.5$ and the number of computational cells with electrostatic field strength commensurate with or stronger than the initial homogeneous magnetic field. A Spearman rank test yields correlation coefficients in the range $0.7-0.9$ for all six simulation runs with very small $p$-values, indicating a good correlation.

\subsubsection{Electron Acceleration (Run C2)}

The main stages of electron acceleration for the out-of-plane magnetic-field configuration (run C2) are shown in Figure 11, in which we trace the trajectory and the energy evolution of a typical particle that becomes energized at the shock. At time $t_{a}$ the electron resides in the Buneman-wave region and is subjected to the SSA process, through which its energy reaches $\gamma \simeq 2.5$. In this example, the electron does not experience another SSA cycle, but starts to gyrate around the mean magnetic field and passes through the shock ramp towards the overshoot. The projection of its gyromotion on the convective electric field in the shock ramp imposes quasi-sinusoidal variations in its kinetic energy. A significant energy increase arises only beyond time $t_{b}$, when the electron approaches the overshoot. The highest energy of $\gamma \simeq 6$ is reached around the time of the highest compression in the overshoot, shortly after $t_{c}$, and upon entering the downstream region, in which the plasma compression is much smaller than in the overshoot and the energy of the electron slowly decreases. The acceleration beyond the SSA phase is thus largely adiabatic. In fact, after time $t=t_{a}$, the average value of the magnetic moment of the electron remains constant (compare Amano \& Hoshino 2009a).

To examine the dynamics of the acceleration processes, we follow the temporal evolution of the spectrum for a selected portion of upstream electrons traversing the shock structure. Figure 12(a) displays electron spectra at four points in time, and panels (b)-(e) indicate the location of the particles for each of the four instances. Each spectrum is fitted with a relativistic Maxwellian (dashed lines in Figure 12(a)), and the fraction of nonthermal electrons is estimated. A suprathermal tail is evident in the spectra already after passage though the Buneman-wave field at the shock foot (Figure 12(b)). During 

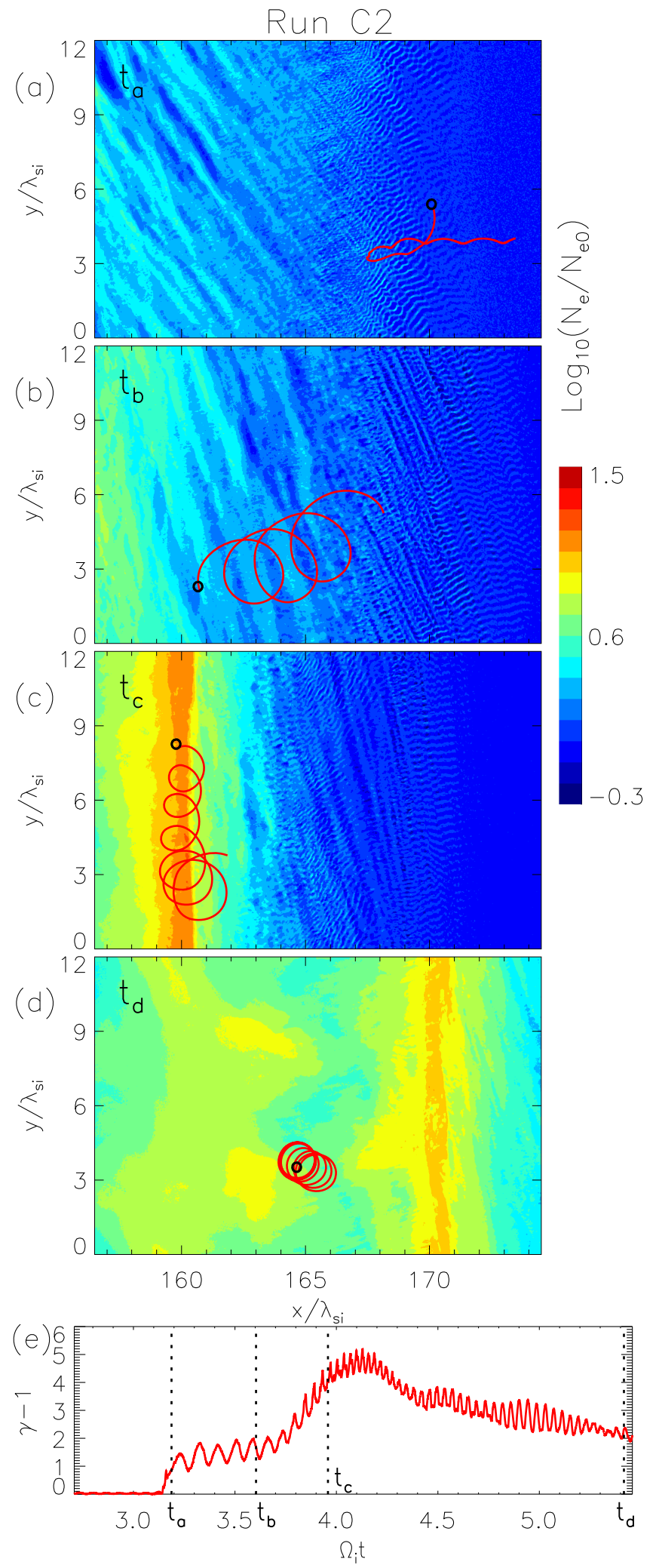

Figure 11. Trajectory segments of a high-energy electron from run C2 superposed on the electron density map at four time intervals. The panels (a)-(d) display the state of the system at the times $t_{a}$ (a), $t_{b}(\mathrm{~b}), t_{c}$ (c), and $t_{d}$ (d) that are marked in panel (e), and the black circles indicate the position of the electron at these moments. The red lines give their trajectory history for the time span $0.3 \Omega_{i}^{-1}$. The color scale for the normalized electron density is logarithmic. In panel (e) we present the temporal development of the kinetic energy of the electron.
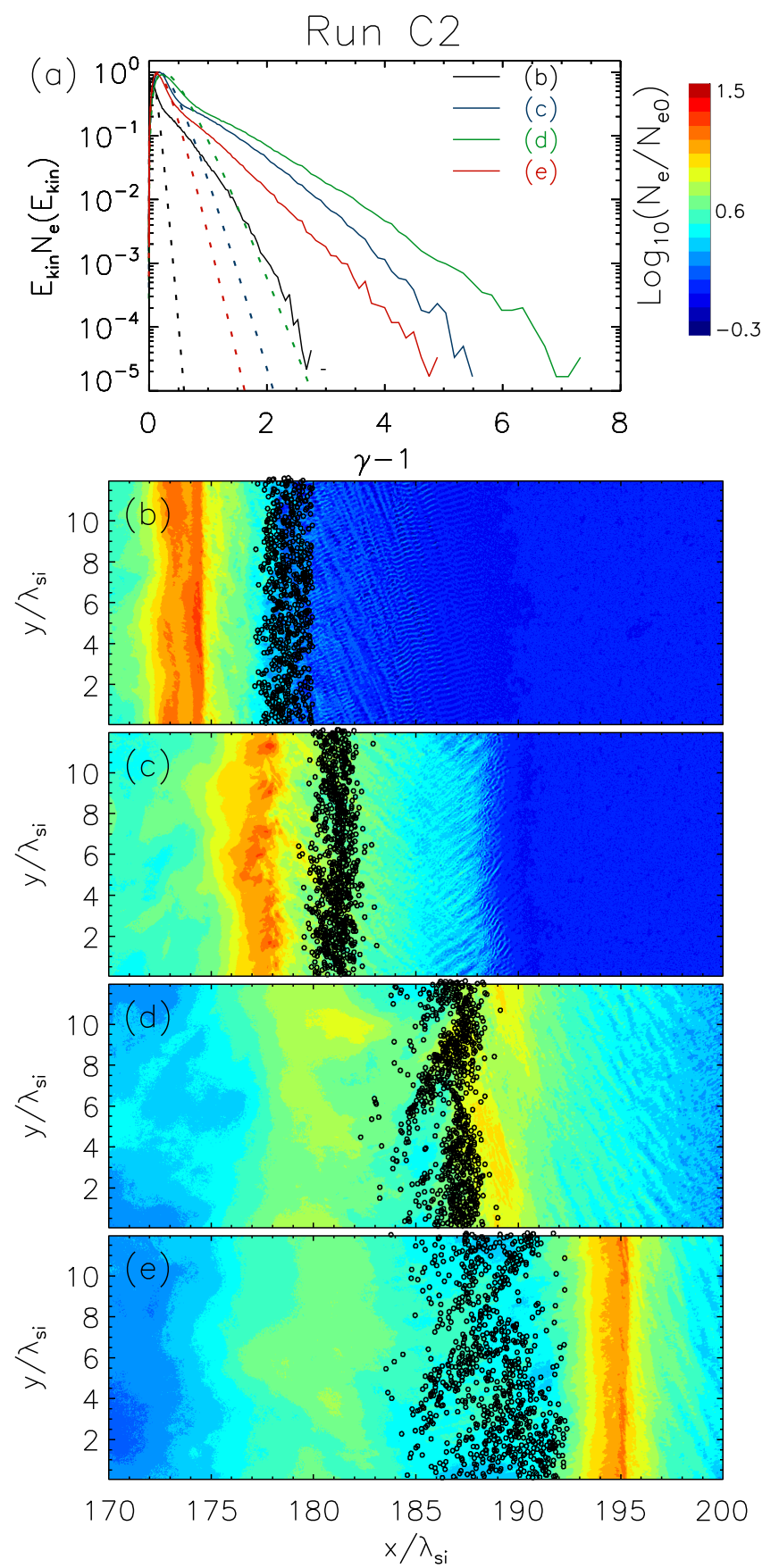

Figure 12. Panel (a): spectral evolution of electrons extracted from the downstream region for run $\mathrm{C} 2$ compared to fits of a relativistic Maxwellian plotted as dotted lines. Panels (b)-(e) present density maps of the shock region together with the positions of electrons selected in the upstream region. The panels display the status at time $t=5.625 \Omega^{-1}, 6.25 \Omega^{-1}, 7 \Omega^{-1}$, and $7.375 \Omega^{-1}$, respectively. The logarithmic color legend for the density maps is placed near panel (a).

their further transport through the shock, the electrons are accelerated to very high energies, and at the same time their bulk temperature increases. The nonthermal electron fraction is about $4.8 \pm 0.2 \%$, carrying $\sim 26 \%$ of the total electron energy. It remains roughly constant once the particles pass through the overshoot and propagate toward the downstream region, where the bulk temperature decreases and the spectral tails become less prominent than those at the overshoot. This behavior provides general support for the notion that the acceleration 
beyond the SSA phase is adiabatic, as we demonstrated for a single particle above. Particle heating is achieved through bulk plasma compression in the shock that is strongest at the overshoot.

\subsubsection{Electron Acceleration (Run A2)}

Electron acceleration in the case of the in-plane magneticfield configuration (run A2) has been analyzed in a similar way as for the out-of-plane field (Section 3.4.2). Figure 13 follows the trajectory and the kinetic-energy history of a typical particle acquiring high energy in interactions within the shock structure, and Figure 14 displays spectra of a selected electron population traversing the shock. Note that for the in-plane magnetic field the SSA process in the shock foot results in moderate particle energization. The Lorentz factor of the electron increases only to $\gamma \simeq 1.5$ while it resides in the Buneman zone at the shock foot (time $t_{a}$ in Figure 13(a)), and the suprathermal tail in the spectrum in Figure 14(a) does not reach $\gamma=2$. Subsequent acceleration (Figures 13(b)-(d)) in the shock structure is markedly different from that observed in the case of the out-of-plane magnetic field: the particle interacts many times with moving magnetic structures, essentially undergoing a stochastic (second-order Fermi) acceleration processes. Its energy increases steadily, and the rate of the energy gain is larger in the shock overshoot region (between times $t_{c}$ and $t_{d}$ in Figure 13(e)) than in the shock ramp (with a time range approximately from $t_{b}$ to $t_{c}$ in Figure 13(e)), because the turbulent magnetic field is stronger at the overshoot. In the end, the sample electron reaches a maximum energy of about $\gamma \simeq 9$, and it retains that energy while it is advected into the downstream region of the shock. Electron injection for the inplane and the $\varphi=45^{\circ}$ field configuration thus mainly involves irreversible non-adiabatic acceleration processes.

The observation of non-adiabatic acceleration is supported by an analysis of the electron spectra in Figure 14 that remain largely unchanged once particles reach their maximum energies and are transmitted downstream (Figures 14(d)-(e)). Small differences in these spectra may result in part from the plasma decompression behind the overshoot, but most probably they reflect the shock reformation. Note that the scattering of energetic particles is accompanied by significant bulk particle heating. As a result, the fraction of nonthermal electrons in the downstream spectrum is about an order of magnitude less than that obtained in the $\varphi=90^{\circ}$ case. The spectra also decay at smaller energies, compared with the case of run $\mathrm{C} 2$.

There is some uncertainty in the fraction of nonthermal electrons, because during the shock transition the particles disperse, and so electrons initially confined in a narrow range of $x$ coordinates are distributed over an $x$-range of $10 \lambda_{\text {si }}$ once they are in the downstream region, in particular for run $\mathrm{C} 2$ $\left(\varphi=90^{\circ}\right)$. The nonthermal fractions are calculated using the Maxwellian fits to the low-energy spectra that are presented in Figures 12(a) and 14(a). We find 8.6\%, 6.4\%, 5.9\%, and 4.8\% for the snapshots (b)-(e) in Figure 12(a); i.e., the out-of-plane configuration, whereas for run $\mathrm{A}$ and an in-plane magnetic field we obtain $0.67 \%, 0.21 \%, 0.31 \%$, and $0.41 \%$ (for snapshots (b)-(e) in Figure 14(a)). The increase in the nonthermal fraction between the shock ramp, the overshoot, and the downstream region of the simulation with the in-plane configuration (run A) reflects the non-adiabatic acceleration processes that appear to operate near the overshoot. In contrast, very efficient acceleration by SSA is observed in the shock foot for out-of-plane
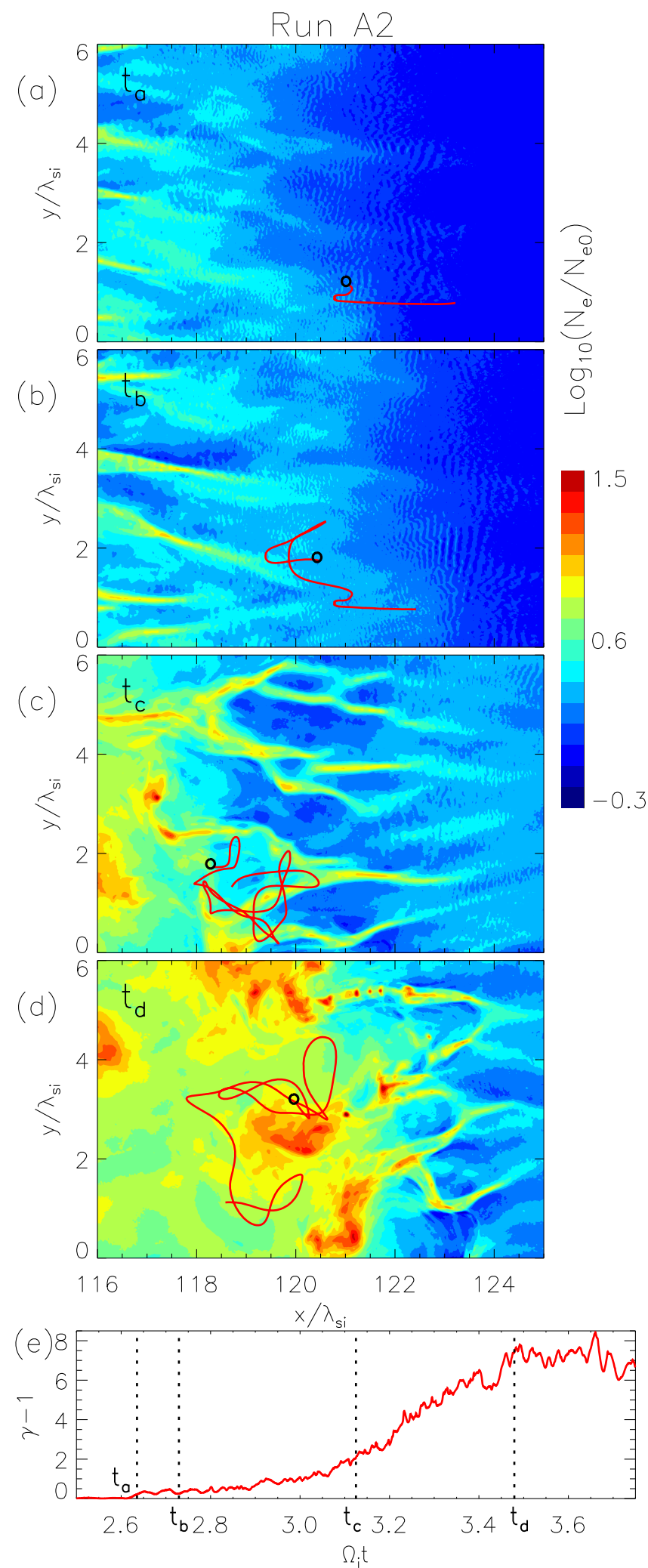

Figure 13. Trajectory segments of a high-energy electron from run A2 superposed on an electron-density map at four time intervals. In panel (e) we present the temporal development of the kinetic energy of the electron and mark four points in time. Panels (a)-(d) display the state of the system at these times $\left(t_{a}(\mathrm{a}), t_{b}(\mathrm{~b}), t_{c}(\mathrm{c})\right.$, and $\left.t_{d}(\mathrm{~d})\right)$, and the black circles indicate the position of the electron at these moments. The red lines give the trajectory history for the time span $0.2 \Omega_{i}^{-1}$. The color scale for the normalized electron density is logarithmic. 

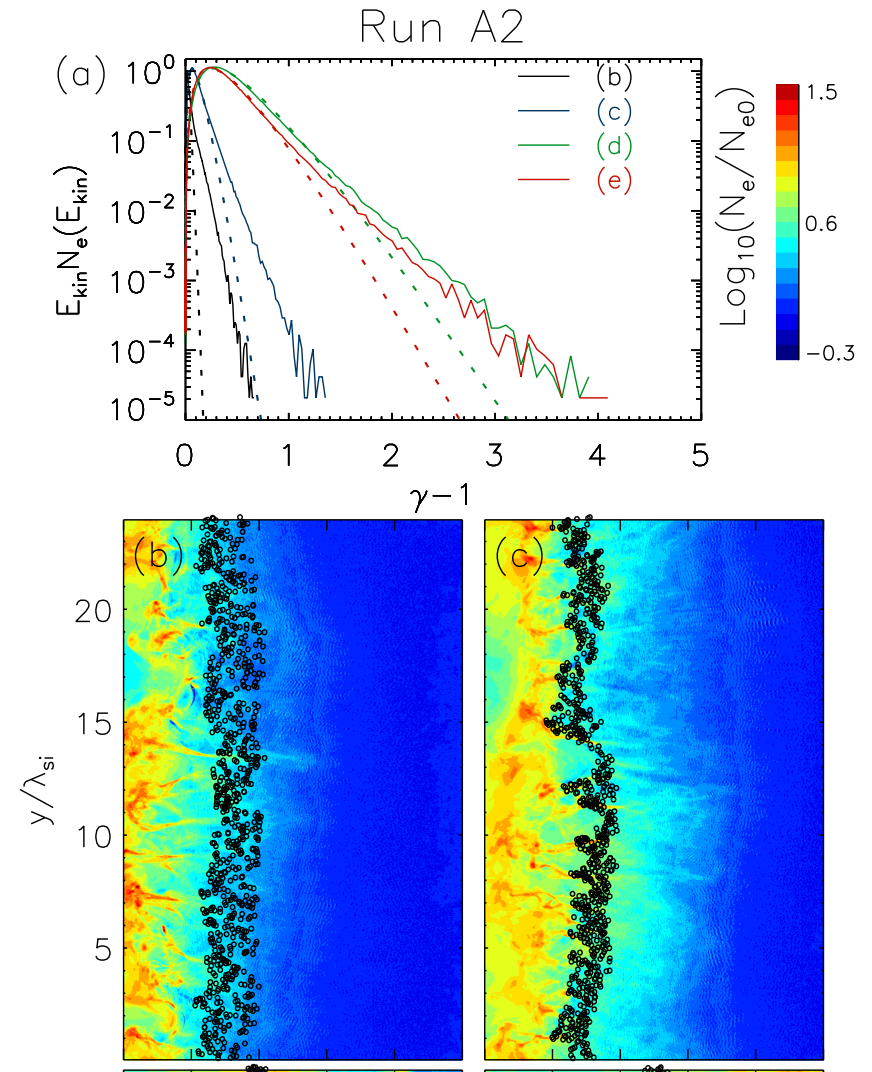
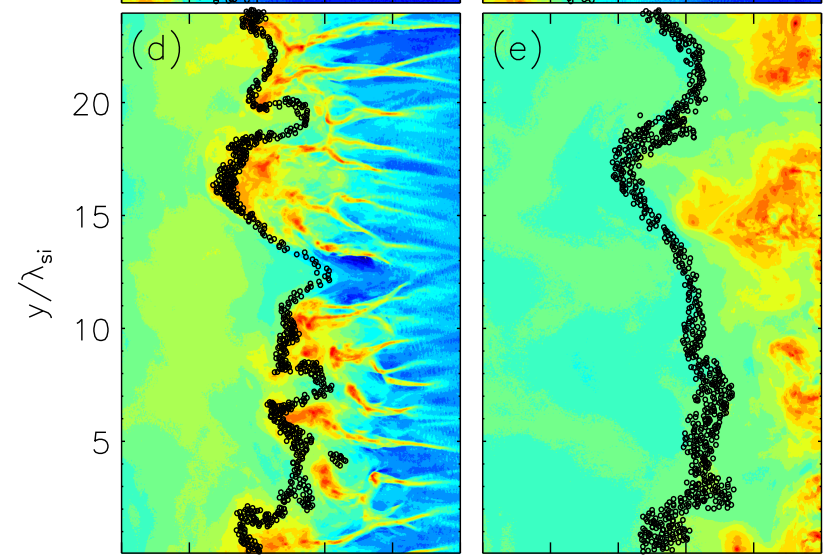

139142145148151

$$
\times / \lambda_{\text {si }}
$$

139142145148151

$$
x / \lambda_{\text {si }}
$$

Figure 14. Panel (a) displays the spectral evolution of electrons selected in the downstream region for run A2. The dotted lines indicate adaptations of a relativistic Maxwellian. Panels (b)-(e) present density maps of the shock region and the positions of the selected electrons at the time $t=5.75 \Omega^{-1}, 5.875 \Omega^{-1}$, $6.625 \Omega^{-1}$, and $7.375 \Omega^{-1}$. Density is presented in logarithmic scale, and the color legend is placed near panel (a).

magnetic field, and in the shock ramp and at the overshoot we loose nonthermal energy by randomization and heating.

The shocks in the B runs (for $\varphi=45^{\circ}$ ) essentially behave like those in the simulations with the in-plane magnetic field (A runs), and so the contents of this subsection also apply to the shocks in $\mathrm{B}$ runs.

\subsubsection{The Influence of Shock Reformation}

In Section 3.2 we discussed the significant influence of the cyclic self-reformation on the structure and speed of the shock, the intensity of Buneman waves, and subsequently on particle
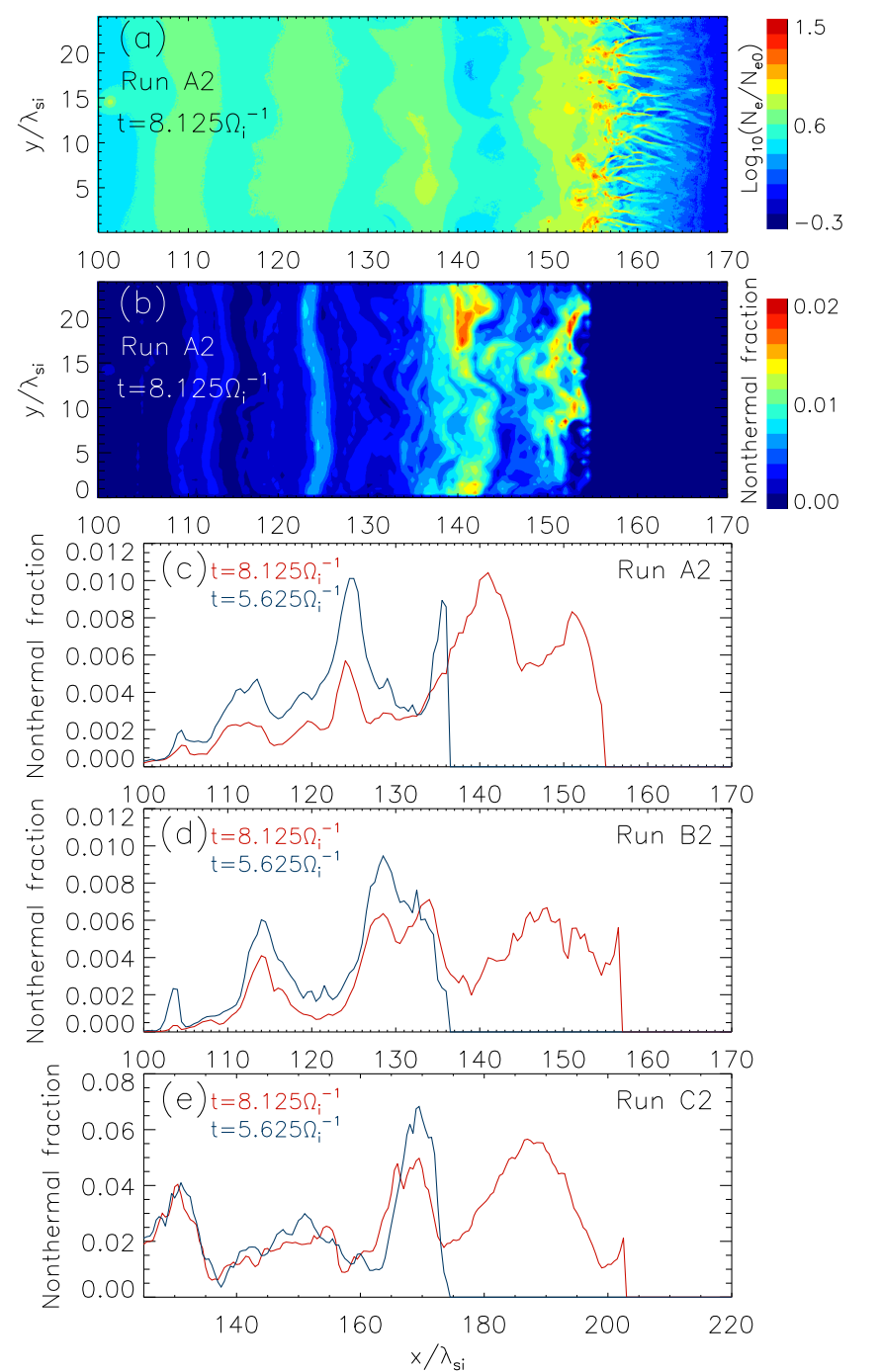

Figure 15. Normalized electron density in logarithmic scale (a) and nonthermal electron fraction (b) for run A2 at time $t=8.125 \Omega_{i}^{-1}$. The three lower panels display profiles of nonthermal electron fraction at times $t=5.625 \Omega_{i}^{-1}$ (blue) and at $t=8.125 \Omega_{i}^{-1}$ (red) for run $\mathrm{A} 2(\mathrm{c})$, run B2 (d), and run $\mathrm{C} 2$ (e).

acceleration. Another consequence is that the downstream particle distributions are not uniform, and the choice of region matters from which we extract particle spectra.

In the downstream region, even in small regions of size $0.5 \lambda_{\mathrm{si}} \times 0.5 \lambda_{\mathrm{si}}$ we find a large number of computational particles (on average $2 \times 10^{5}$ for runs A2/B2 and $1.5 \times 10^{5}$ for run $\mathrm{C} 2$ ). Hence, local electron spectra can be calculated with reasonable precision. Likewise, we can evaluate the local temperature by fitting a relativistic Maxwellian and determine the fraction of nonthermal electrons. Figure 15 shows maps of the electron density and nonthermal electron fraction in the downstream region of the moderate- $\beta_{\mathrm{p}}$ shock in run A2. Note the inhomogeneous structure of the downstream region that results from shock reformation. Regions of higher density are signatures of the reformation phases at which the shock overshoot had the highest density. The nonthermal electron fraction is also nonuniform (Figure 15(b)). The simulation is too short to permit homogenization of the downstream region, implying that it will be achieved only very far downstream.

Also displayed in Figure 15 are profiles of the nonthermal electron fraction for all moderate- $\beta_{\mathrm{p}}$ runs. Note the large 
variations in the nonthermal fraction in the downstream region. They reflect variations in both the bulk temperature and the number of high-energy electrons that do not coincide. In panel (c) we present profiles (averaged over $y$-direction) of the nonthermal electron fraction in run $\mathrm{A} 2$ at times $t=5.625 \Omega_{i}^{-1}$ (blue) and at $t=8.125 \Omega_{i}^{-1}$ (red). One can see four maxima in the nonthermal fraction (around $x / \lambda_{\mathrm{si}} \simeq 112,124,141$, and 152) for the red line that trace back to passage through the most intense electrostatic-wave field in the foot region. The blue line displays the nonthermal fraction at an earlier time $\left(t=5.625 \Omega_{i}\right)$, and we note that the nonthermal fraction at a fixed location was higher at earlier times, indicating a decrease over time of the abundance of pre-accelerated electrons in the downstream region. We observe the same trend for run B, which may explain the marginal nonthermal population found in the far-downstream region in the simulation of Wieland et al. (2016). It is remarkable that we do not observe a similar loss of nonthermal electrons in the simulation with the out-of-plane magnetic field (Figure 15(e)). There the amplitude of variations in temperature and electron density is also larger and somewhat correlated, which suggests that homogenization is not as efficient as for runs $\mathrm{A}$ and $\mathrm{B}$.

\subsubsection{Spectra in the Downstream Region}

We conclude the presentation of our results with the energy spectra of electrons in the downstream region. For that purpose we chose a region behind the overshoot that contains particles processed over two cycles of the shock reformation. The extent of this region is $2 \times 1.55 \Omega_{i}^{-1} \times v_{\mathrm{sh}} \simeq 25 \lambda_{\mathrm{si}}$ for runs $\mathrm{A} / \mathrm{B}$ and $37.5 \lambda_{\mathrm{si}}$ for run $\mathrm{C}$ (recall that the shock speed is higher in run $\mathrm{C}$ ).

Electron spectra for the in-plane $\left(\varphi=0^{\circ}\right), \varphi=45^{\circ}$, and the out-of-plane $\left(\varphi=90^{\circ}\right)$ configuration of the magnetic field and $\beta_{\mathrm{p}}=0.5$ are presented in Figure 16(a). In all of the simulations we observe electrons with Lorentz factors up to $\gamma \approx 9$. The main difference between the spectra is at low energies, where we can fit relativistic Maxwellians to represent the bulk of electrons, shown here with dashed lines. Note the variation in the plasma temperature that results from the choice of the magnetic-field configuration. In the lower panel (b) of Figure 16 we display spectra in energy scaled to the plasma temperature. Whereas for $\varphi=0^{\circ}$ and $45^{\circ}$ we find almost indistinguishable spectra in rescaled energy, those for run $\mathrm{C}$ with the out-of-plane field feature a much more pronounced spectral tail.

Table 2 summarizes our findings: the downstream temperature, the nonthermal electron fraction, and the number of energetic electrons are presented for all runs. Note that beside the fit uncertainty in the temperature, there is a spatial variation of plasma temperature in the downstream region, and so we consider the plasma temperature for low and moderate $\beta_{\mathrm{p}}$ the same within the uncertainties. As was shown in Sections 3.4.1-3.4.3, the number of pre-accelerated electrons and the final abundance of nonthermal electrons depends on the efficiency of acceleration by the Buneman waves. The electron temperatures are higher by a factor 2-4 than those predicted by the Rankine-Hugoniot jump conditions, indicating that significant bulk heating has occurred (compare Matsumoto et al. 2012).

It is remarkable that there is no direct relation between largeamplitude electrostatic field and the fraction of nonthermal electrons. While the uncertainties in the determination are sizable, we find a higher nonthermal fraction for moderate- $\beta_{\mathrm{p}}$
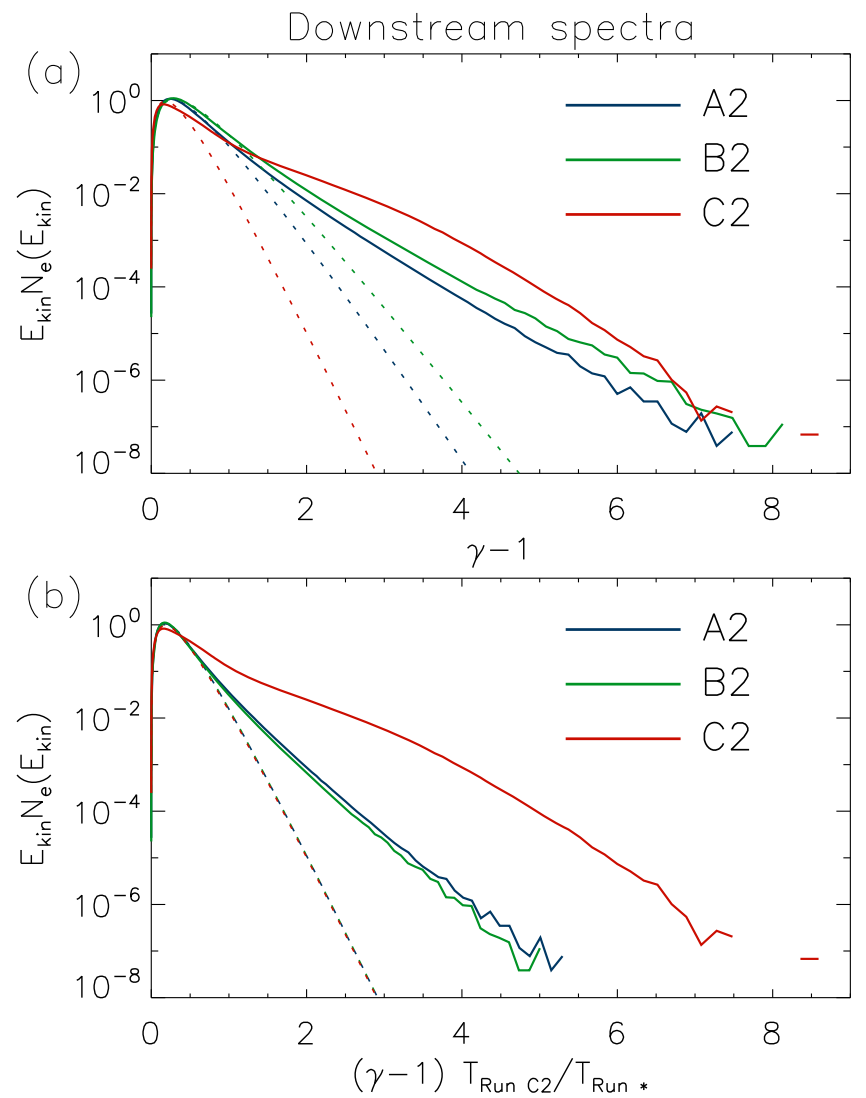

Figure 16. In the top panel (a) we show electron spectra in the downstream region of the moderate- $\beta_{\mathrm{p}}$ shocks. The dashed lines represent fits of a relativistic Maxwellian to the low-energy spectra. The bottom panel (b) displays the distribution in rescaled energy in units of the downstream temperature. Blue lines correspond to run $\mathrm{A} 2$, green curves to run $\mathrm{B} 2$, and red curves to run $\mathrm{C} 2$.

Table 2

Downstream Spectra Parameters

\begin{tabular}{lclccc}
\hline \hline Run & $\varphi$ & $\beta_{\mathrm{p}}$ & NTEF $(\%)$ & $N_{e}(\gamma>3)(\%)$ & $k T / m c^{2}$ \\
\hline A1 & $0^{\circ}$ & 0.0005 & $0.2 \pm 0.1$ & 0.1 & 0.053 \\
A2 & $0^{\circ}$ & 0.5 & $0.7 \pm 0.1$ & 0.06 & 0.043 \\
\hline B1 & $45^{\circ}$ & 0.0005 & $0.2 \pm 0.1$ & 0.11 & 0.054 \\
B2 & $45^{\circ}$ & 0.5 & $0.5 \pm 0.1$ & 0.1 & 0.049 \\
\hline C1 & $90^{\circ}$ & 0.0005 & $4 \pm 1$ & 0.12 & 0.032 \\
C2 & $90^{\circ}$ & 0.5 & $7 \pm 1$ & 0.3 & 0.03 \\
\hline
\end{tabular}

Note. Comparison of the characteristics of the energy distribution of electrons in the downstream region of all six simulated shocks. NTEF denotes the nonthermal electron fraction.

shocks, whatever the orientation of the large-scale magnetic field.

We do observe a correlation between the abundance of strong electrostatic field and the presence of a high-energy tail in the final downstream spectrum, expressed as either the maximum energy or the number of high-energy electrons with $\gamma>3$. For the number of high-energy electrons one can find the same trend in all simulations: if the number of grid points with high-amplitude electrostatic field, $N_{\mathrm{es}}\left(E_{\mathrm{es}}>0.5 B_{0}\right)$, is high, then the number of high-energy electrons, $N_{e}(\gamma>3)$, is also high. Comparing shocks propagating in the cold and the 
warm plasma (low and moderate $\beta_{\mathrm{p}}$, respectively), we find for run A $N_{\text {es,cold }}\left(E_{\text {es }}>0.5 B_{0}\right)>N_{\text {es,warm }}\left(E_{\text {es }}>0.5 B_{0}\right)$, and indeed we observe a higher abundance of high-energy electrons for the low- $\beta_{\mathrm{p}}$ shock. For run $\mathrm{B}$, both the abundance of intense electrostatic field and the spectral tails are similar for low and moderate $\beta_{\mathrm{p}}$. For run $\mathrm{C}$, the correlation holds, but now we find strong electric field more rarely at shocks propagating into the cold plasma, and there are fewer energetic particles there than at the moderate- $\beta_{\mathrm{p}}$ shock. We can conclude that the population size of high-energy electrons (but not necessarily the nonthermal fraction of electrons) is determined by energization in the Buneman zone.

Efficient electron acceleration during passage through the shock ramp and overshoot will weaken the relation between the nonthermal fraction of electrons and the efficiency of SSA. In the $\varphi=90^{\circ}$ case with the out-of-plane magnetic field, the electron transport beyond the Buneman zone is adiabatic. Consequently, the distribution of electrons in magnetic moment (instead of their energy) is constant in time, as is the numerical relation between thermal and nonthermal electrons. Then the number of nonthermal electrons should be a direct tracer of the SSA efficiency. In the $\varphi=0^{\circ}$ and $\varphi=45^{\circ}$ configurations, second-order Fermi-like processes operating in the shock ramp can change the number ratio of the thermal bulk and the nonthermal population. Further studies are needed to explore the nature of non-adiabatic acceleration processes, for example spontaneous turbulent magnetic reconnection (Matsumoto et al. 2015), for which the shock reformation provides an additional complication. We defer this issue to a further publication.

\section{Summary and Discussion}

In this paper we present results of our 2D3V PIC simulations of electron injection and acceleration processes in nonrelativistic perpendicular collisionless shocks with high Alfvénic Mach numbers. The simulation parameters are chosen to permit the growth of the Buneman waves and the trapping of electrons at the shock foot (see Matsumoto et al. 2012). We explored the efficiency of electron energization for shocks in plasmas with low and moderate $\beta_{\mathrm{p}}$ and for three orientation angles, $\varphi$, between the large-scale magnetic field and the simulation plane. The Alfvénic Mach number is $M_{\mathrm{A}}=31.8$ for simulations with angle $\varphi=0^{\circ}$ and $45^{\circ}$, whereas for $\varphi=90^{\circ}$ we have $M_{\mathrm{A}}=35.6$ on account of a larger effective adiabatic index. We follow the temporal evolution of shock structures for eight ion gyrotimes, $t=8 \Omega_{i}^{-1}$, to study the influence of the shock selfreformation on particle acceleration.

Our results can be summarized as follows.

1. The collision of two plasma slabs leads to the formation of a double shock system and a CD, and the development time of the shocks is about $2 \Omega_{i}^{-1}$. In general, the structure of the shocks is similar in all runs; they consist of the upstream, foot, overshoot, and downstream regions. In detail, the influence of the global magnetic field on the phase-space distribution of shock-reflected ions imposes differences in the evolution of the Buneman and the Weibel instability. Magnetic reconnection events are observed at the Weibel-mode zone in simulations with $0^{\circ}$ and $45^{\circ}$ magnetic-field orientations.

2. The simulated shocks undergo cyclic self-reformation on account of the nonstationary character of ion reflection at the shock. The period of shock reformation is $\sim 1.55 \Omega^{-1}$, which is close to the value obtained in our earlier investigations $\left(1.5 \Omega^{-1}\right.$ in Wieland et al. 2016). This period depends neither on the magnetic-field configuration nor on the plasma beta.

3. Reflected ions interact with incoming electrons and excite electrostatic Buneman waves at the leading edge of the foot region. The parameters of our simulations satisfy the trapping condition (Matsumoto et al. 2012), and so electrons can be accelerated by the convective electric field. The intensity of the electrostatic waves, the area they occupy, and, subsequently, the fraction of nonthermal electrons strongly depend on the global magnetic-field orientation. Simulations with the out-of-plane magneticfield configuration produce a much higher fraction of nonthermal electrons. In the cases $\varphi=0^{\circ}$ and $\varphi=45^{\circ}$, part of the counter-streaming of reflected ions proceeds in $z$ direction, but the $k_{z}$ component of the Buneman waves driven by it are not captured by the $x-y$ simulation grid. Consequently, the saturation level of the waves and their ability to trap electrons is less than for $\varphi=90^{\circ}$, for which we observe a higher intensity of Buneman waves at the shock foot and a larger volume coverage. With Equation (6) we propose a revision to the trapping condition originally formulated by Matsumoto et al. (2012) that offers an explanation as to why some of the simulation presented here, and others described in the literature, have a low Buneman efficiency. If the new revised trapping condition (6) is met, as in our simulation with $\varphi=90^{\circ}$, then the number of electrons undergoing SSA is large, at least as long as the plasma beta $\beta_{\mathrm{p}} \lesssim 1$. A similar modification can be applied to the driving condition, leading to Equation (7).

4. Shock self-reformation leads to temporal variations in the electrostatic-wave intensity in the foot region, and consequently the electron energization in these regions becomes time-dependent. After crossing the Bunemanwave zone the fraction of nonthermal electrons does not change significantly. The cyclic shock reformation then imposes quasi-periodic fluctuations in the temperature and density of bulk electrons as well as the density of high-energy electrons, which leads to variations in the nonthermal fraction of electrons. The amplitude of these variations is largest for out-of-plane magnetic field, where we also observe spatial correlations between the temperature and the density of bulk electrons that render homogenization of downstream electrons a slow process. That is not the case for runs with $\varphi=0^{\circ}$ and $\varphi=45^{\circ}$, and consequently we observe the fraction of nonthermal electrons in the downstream region decreasing with time.

5. For all simulated shocks we find suprathermal tails in the electron spectra in the downstream region, but the acceleration efficiency depends on the magnetic-field orientation. For all configurations the main acceleration process is through interaction with electrostatic waves in the Buneman-wave zone at the shock foot, followed by further energization by turbulent magnetic structures and in the shock overshoot. The weight of the individual contributions by all of these processes depends on the magnetic-field configuration. The cases $\varphi=0^{\circ}$ and $\varphi=45^{\circ}$ lead to the same acceleration processes, and we do not see any significant difference between them. By contrast, the $\varphi=90^{\circ}$ configuration provides a 
Table 3

Comparison of the Electron Pre-acceleration Efficiency in the Present and in Other Published Simulations

\begin{tabular}{|c|c|c|c|c|c|c|}
\hline Run & $\varphi$ & $\beta_{\mathrm{p}}$ & Equation (2) & Equation (6) & Nonthermal Population & Notes \\
\hline $\mathrm{A} 1 ; \mathrm{A} 2$ & $0^{\circ}$ & $0.0005 ; 0.5$ & Yes & No & Weak & \\
\hline Kato \& Takabe (2010) & $0^{\circ}$ & 26 & Yes & Yes & Absent & High temperature of reflected ions \\
\hline Matsumoto et al. (2015) & $0^{\circ}$ & 0.5 & Yes & No & Weak or absent & \\
\hline B1; B2 & $45^{\circ}$ & $0.0005 ; 0.5$ & Yes & No & Weak & \\
\hline Wieland et al. (2016) & $45^{\circ}$ & $0.0015 ; 0.015$ & Yes & Yes & Weak or absent & Spectra far downstream \\
\hline $\mathrm{C} 1 ; \mathrm{C} 2$ & $90^{\circ}$ & $0.0005 ; 0.5$ & Yes & Yes & Strong & \\
\hline Matsumoto et al. (2012) (Run A) & $90^{\circ}$ & 0.5 & Yes & Yes & Strong & \\
\hline Matsumoto et al. (2012) (Run B) & $90^{\circ}$ & 0.5 & No & No & Weak & \\
\hline Matsumoto et al. (2012) (Run C) & $90^{\circ}$ & 0.5 & Yes & Yes & Strong & \\
\hline Matsumoto et al. (2012) (Run D) & $90^{\circ}$ & 4.5 & Yes & Yes & Weak & Weak driving \\
\hline
\end{tabular}

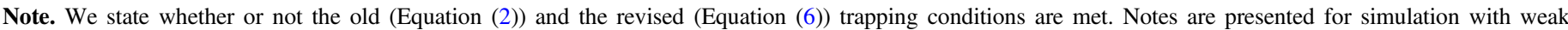
nonthermal population and where both old (Equation (2)) and the revised (Equation (6)) trapping conditions are satisfied.

fundamentally different behavior. In this case the intensity of Buneman waves at the shock foot is higher, because the new trapping condition (Equation (6)) is satisfied, and the waves are coherent and found in a larger region as the result of coherent ion reflection by magnetic field at the overshoot. One consequence is that electrons gyrating in the foot region can cross the Buneman-wave zone more than once, experience more SSA, and reach a higher energy than they would with a $\varphi=0^{\circ}$ or $\varphi=45^{\circ}$ configuration, for which we do not observe such multiple interactions. The number of high-energy electrons (at $\gamma>1.5)$ in the Buneman zone is correspondingly larger for $\varphi=90^{\circ}$ than it is for the other cases. In all of the simulations the Buneman instability serves as an injector; i.e., electrons are accelerated to suprathermal energies by electrostatic waves at the leading edge of the foot region. The second stage of electron energization is second-order Fermi acceleration by interaction with magnetic filaments in simulations with a $0^{\circ}$ or $45^{\circ}$ configuration, and it is adiabatic acceleration in the case of the out-of-plane magnetic field. For all simulations, the Lorentz factor of the most energetic electron is $\gamma \approx 9$, but the fraction of nonthermal electrons is more then 10 times larger for $\varphi=90^{\circ}$ than for the other configuration on account of the higher SSA efficiency in the foot region. There is no clear trend between the number of high-energy electrons and the $\beta_{\mathrm{p}}$ value of the plasma into which the shock propagates. For $\varphi=90^{\circ}$, we observe more electrons above $\gamma=3$ in the moderate- $\beta_{\mathrm{p}}$ case than for the low $\beta_{\mathrm{p}}$. For the other simulations that have at least part of the large-scale magnetic field in the simulation plane, we find the opposite trend. This issue will be the subject of a separate publication. However, for a given magnetic-field orientation we observe a higher nonthermal fraction at shocks propagating into moderate- $\beta_{\mathrm{p}}$ plasma than for low $\beta_{\mathrm{p}}$.

This paper presents evidence for significant variation in the efficiency of electron acceleration at perpendicular high-Machnumber shocks, depending on the choice of orientation of the large-scale magnetic field with respect to the simulation plane. Much, but not all, of the variation can be traced to the efficiency of driving the Buneman instability at the shock foot. Our findings are summarized in Table 3 in the context of other published results (Kato \& Takabe 2010; Matsumoto et al. 2012,
2015; Wieland et al. 2016). There are three parameters that have an influence on downstream spectra and the final fraction of nonthermal electrons: the plasma beta, $\beta_{\mathrm{p}}$, the trapping condition in the earlier (Equation (2)) and in the revised form (Equation (6)), and the driving condition that can be likewise revised (Equation (7)). For the $\varphi=90^{\circ}$ case the old and new trapping conditions are identical, and the nonthermal electron population appears to be sparse if the trapping condition is not satisfied (run B in Matsumoto et al. 2012) or if a high plasma beta leads to early saturation the Buneman instability (the thermal velocity of electrons is about half of the streaming speed of reflected ions in run D of Matsumoto et al. 2012). A weak population of nonthermal electrons is observed in our runs with $\varphi=0^{\circ}$ and $\varphi=45^{\circ}$, as well as in the simulation described by Matsumoto et al. (2015), because of the Alfvénic Mach number is too low to satisfy the revised trapping condition. Wieland et al. (2016) discuss shocks with Mach numbers $M_{\mathrm{A}}$ and $M_{s}$ large enough to drive Buneman waves and trap electrons, even if the modified conditions are applied. Electron spectra are extracted in the far-downstream region where leakage of nonthermal electrons is observed (see Section 3.4.4), which may explain why very few energetic electrons were observed. The modified driving and trapping conditions were also met in the simulation of Kato \& Takabe (2010), where $M_{\mathrm{A}}$ is large enough to satisfy the modified trapping condition, but the authors argue that the high temperature of reflected ions reduced the Buneman growth rate by about an order of magnitude. Further studies of the nonuniformity of ion reflection from a corrugated overshoot structure are needed to confirm this explanation.

We have presented the results of the $2 \mathrm{D} 3 \mathrm{~V}$ simulations, but the real world is 3D throughout. The question arises: which behavior would one observe in $3 \mathrm{D}$ and which of the $2 \mathrm{D} 3 \mathrm{~V}$ configurations provides the closest match to the 3D case? By definition, all wave vectors lie in the simulation plane, and so for $\varphi=90^{\circ}$ only electromagnetic modes with $B_{x}$ or $B_{y}$ components can rotate particles out of the simulation plane. Particle trajectories are thus approximately confined to the simulation plane, and the particle ensemble assumes an effective adiabatic index of 2 , as opposed to $5 / 3$ for a $3 \mathrm{D}$ monoatomic gas. We indeed observe the corresponding difference in shock speeds, etc., and the 3D shock structure may be not accurately reproduced for $\varphi=90^{\circ}$. On the other hand, with $\varphi=90^{\circ}$ the counter-streaming of reflected ions and the driving of Buneman modes in the shock foot is fully 
captured by the $x-y$ simulation grid. The fair fraction of nonthermal electrons produced in $\varphi=90^{\circ}$ simulations is probably a better indicator of the $3 \mathrm{D}$ acceleration efficiency at the shock foot than the very low abundance of energetic electrons for $\varphi=0^{\circ}$ and $\varphi=45^{\circ}$. True 3D simulations are urgently needed to resolve this issue.

The work of A.B., J.N., and O.K. is supported by the Narodowe Centrum Nauki through the research project DEC2013/10/E/ST9/00662. M.P. acknowledges support through grants PO 1508/1-1 and PO 1508/1-2 of the Deutsche Forschungsgemeinschaft. Numerical simulations have been performed on the Prometheus system at ACC Cyfronet AGH. Part of the numerical work was also conducted on resources provided by the North-German Supercomputing Alliance (HLRN) under the project bbp00003.

\section{ORCID iDs}

Artem Bohdan (i) https://orcid.org/0000-0002-5680-0766

Jacek Niemiec (ib) https://orcid.org/0000-0001-6036-8569

Oleh Kobzar (iD https://orcid.org/0000-0001-6956-5884

Martin Pohl (i) https://orcid.org/0000-0001-7861-1707

\section{References}

Aharonian, F., Akhperjanian, A. G., Bazer-Bachi, A. R., et al. 2007, A\&A, 464,235

Amano, T., \& Hoshino, M. 2009a, ApJ, 690, 244
Amano, T., \& Hoshino, M. 2009b, PhPl, 16, 102901

Blandford, R., \& Eichler, D. 1987, PhR, 154, 1

Bohata, M., Bren, D., \& Kulhánek, P. 2011, ISRN Condensed Matter Physics, 2011, 896321

Breizman, B. N. 1990, RvPP, 15, 61

Brě̌zman, B. N., \& Ryutov, D. D. 1974, NucFu, 14, 873

Buneman, O. 1958, PhRvL, 1, 8

Buneman, O. 1993, in Computer Space Plasma Physics: Simulation Techniques and Software, ed. H. Matsumoto \& Y. Omura (Tokyo: Terra Scientific), 67 (https://www.terrapub.co.jp/e-library/cspp/)

Burgess, D., \& Scholer, M. 2007, PhPl, 14, 012108

Caprioli, D., \& Spitkovsky, A. 2014, ApJ, 783, 91

Drury, L. O. 1983, RPPh, 46, 973

Furth, H. P., Killeen, J., \& Rosenbluth, M. N. 1963, PhFl, 6, 459

Galeev, A. A., Sagdeev, R. Z., Shapiro, V. D., \& Shevchenko, V. I. 1977, JETP, 45, 266

Hoshino, M., \& Shimada, N. 2002, ApJ, 572, 880

Ishihara, O., Hirose, A., \& Langdon, A. B. 1980, PhRvL, 44, 1404

Kato, T. N., \& Takabe, H. 2010, ApJ, 721, 828

Lembege, B., \& Dawson, J. M. 1987, PhFl, 30, 1767

Matsumoto, Y., Amano, T., \& Hoshino, M. 2012, ApJ, 755, 109

Matsumoto, Y., Amano, T., \& Hoshino, M. 2013, PhRvL, 111, 215003

Matsumoto, Y., Amano, T., Kato, T. N., \& Hoshino, M. 2015, Sci, 347, 974

Niemiec, J., Pohl, M., Bret, A., \& Wieland, V. 2012, ApJ, 759, 73

Niemiec, J., Pohl, M., Stroman, T., \& Nishikawa, K.-I. 2008, ApJ, 684, 1174

Quest, K. B. 1985, PhRvL, 54, 1872

Strangeway, R. J. 1982, JGR, 87, 833

Treumann, R. A. 2009, A\&ARv, 17, 409

Umeda, T., Kidani, Y., Matsukiyo, S., \& Yamazaki, R. 2014, PhPl, 21, 022102

Umeda, T., Yamao, M., \& Yamazaki, R. 2008, ApJL, 681, L85

Umeda, T., Yamao, M., \& Yamazaki, R. 2009, ApJ, 695, 574

Wieland, V., Pohl, M., Niemiec, J., Rafighi, I., \& Nishikawa, K.-I. 2016, ApJ, 820,62 